\title{
Regioisomer-Specific Mechanochromism of Naphthopyran in Polymeric Materials
}

\author{
Maxwell J. Robb, ${ }^{\S, \dagger}$ Tae Ann Kim, ${ }^{\S, \ddagger}$ Abigail J. Halmes, ${ }^{\S, \dagger}$ Scott R. White, ${ }^{\S, \Delta}$ \\ Nancy R. Sottos, ${ }^{*, \$}$ and Jeffrey S. Moore ${ }^{*, \S, \dagger}$ \\ ${ }^{\S}$ The Beckman Institute for Advanced Science and Technology, ${ }^{\dagger}$ Department of Chemistry, \\ ${ }^{\ddagger}$ Department of Materials Science and Engineering, and ${ }^{\triangle}$ Department of Aerospace Engineering, \\ University of Illinois at Urbana-Champaign, Urbana, IL, 61801, USA. \\ •E-mail: jsmoore@illinois.edu; n-sottos@illinois.edu
}

Table of Contents

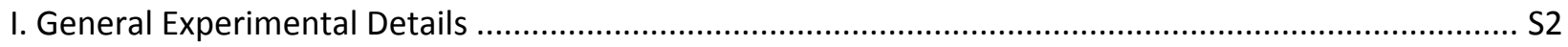

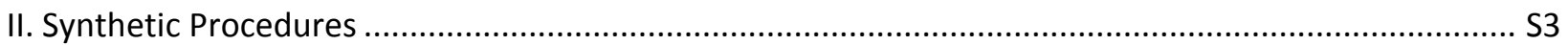

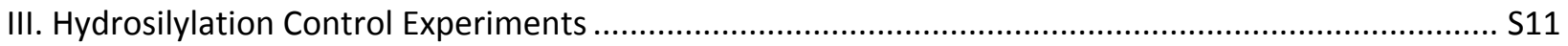

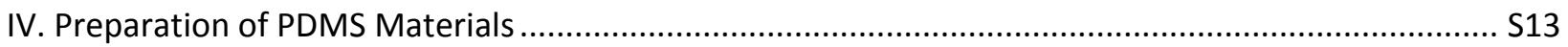

V. In Situ Mechanical/Visible Absorption Measurements ................................................................. S14

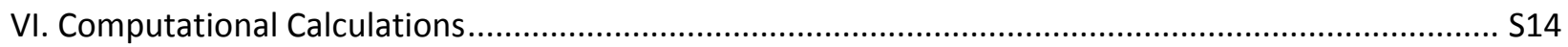

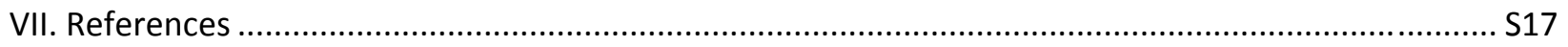

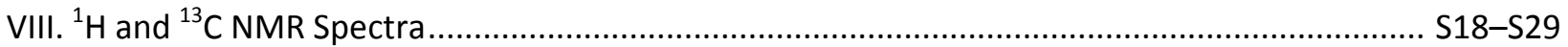



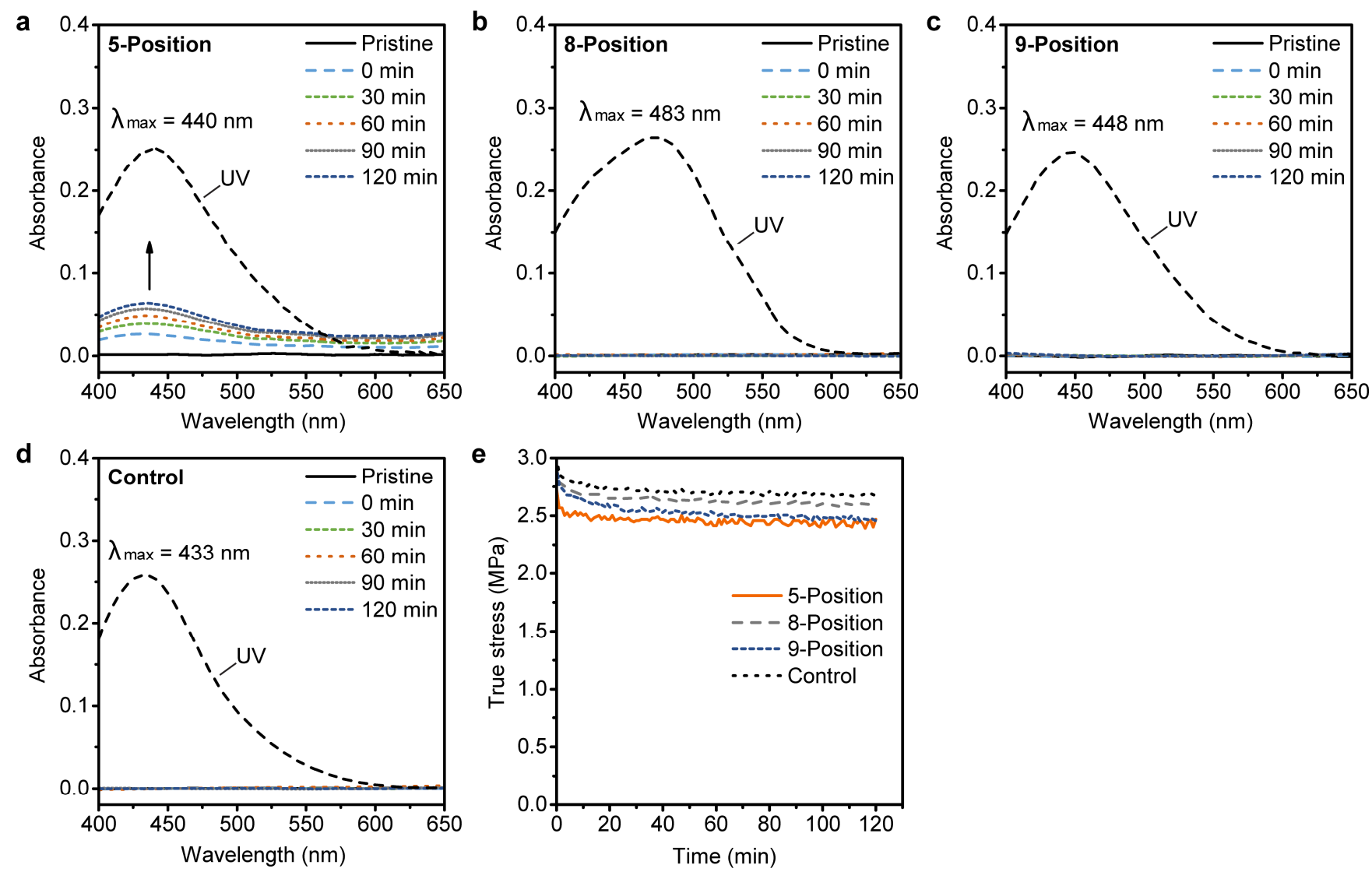

Figure S1. (a-d) Visible absorption spectra of dogbone-shaped PDMS specimens incorporating naphthopyrans under tension and after activation with $365 \mathrm{~nm}$ UV light. Specimens were stretched to a constant value (time $=0 \mathrm{~min}$ ) and held under mechanical stress while absorption spectra were recorded at 30 min intervals. (e) True stress of each specimen during the absorption measurements.

\section{General Experimental Details}

Reagents from commercial sources were used without further purification unless otherwise stated. A two-part polydimethylsiloxane (PDMS) elastomer kit (Dow Corning Sylgard ${ }^{\circledR} 184$ ) was purchased from Fisher Scientific. Dry THF was obtained from a solvent purification system equipped with activated alumina columns and stored over $3 \AA$ molecular sieves. All reactions were performed under a $\mathrm{N}_{2}$ atmosphere unless specified otherwise. Column chromatography was performed on a Biotage Isolera system using SiliCycle SiliaSep HP flash cartridges. NMR spectra were recorded using a Varian $500 \mathrm{MHz}$ spectrometer. All ${ }^{1} \mathrm{H}$ NMR experiments are reported in $\delta$ units, parts per million (ppm), and were measured relative to the signals for residual acetone $(2.05 \mathrm{ppm})$ or DMSO $(2.50 \mathrm{ppm})$ in deuterated 
solvent. All ${ }^{13} \mathrm{C}$ NMR spectra were measured in deuterated solvents and are reported in ppm relative to the signals for residual acetone (206.26 and $29.84 \mathrm{ppm}$ ) or DMSO (39.52 ppm). Mass spectra were obtained through the Mass Spectrometry Laboratory, School of Chemical Sciences, at the University of Illinois. Digital photographs of PDMS materials were acquired using a Canon EOS 7D digital camera $(1 / 250 \mathrm{sec}, \mathrm{f} / 8$, ISO 3200$)$ and are uncorrected.

\section{Synthetic Procedures}
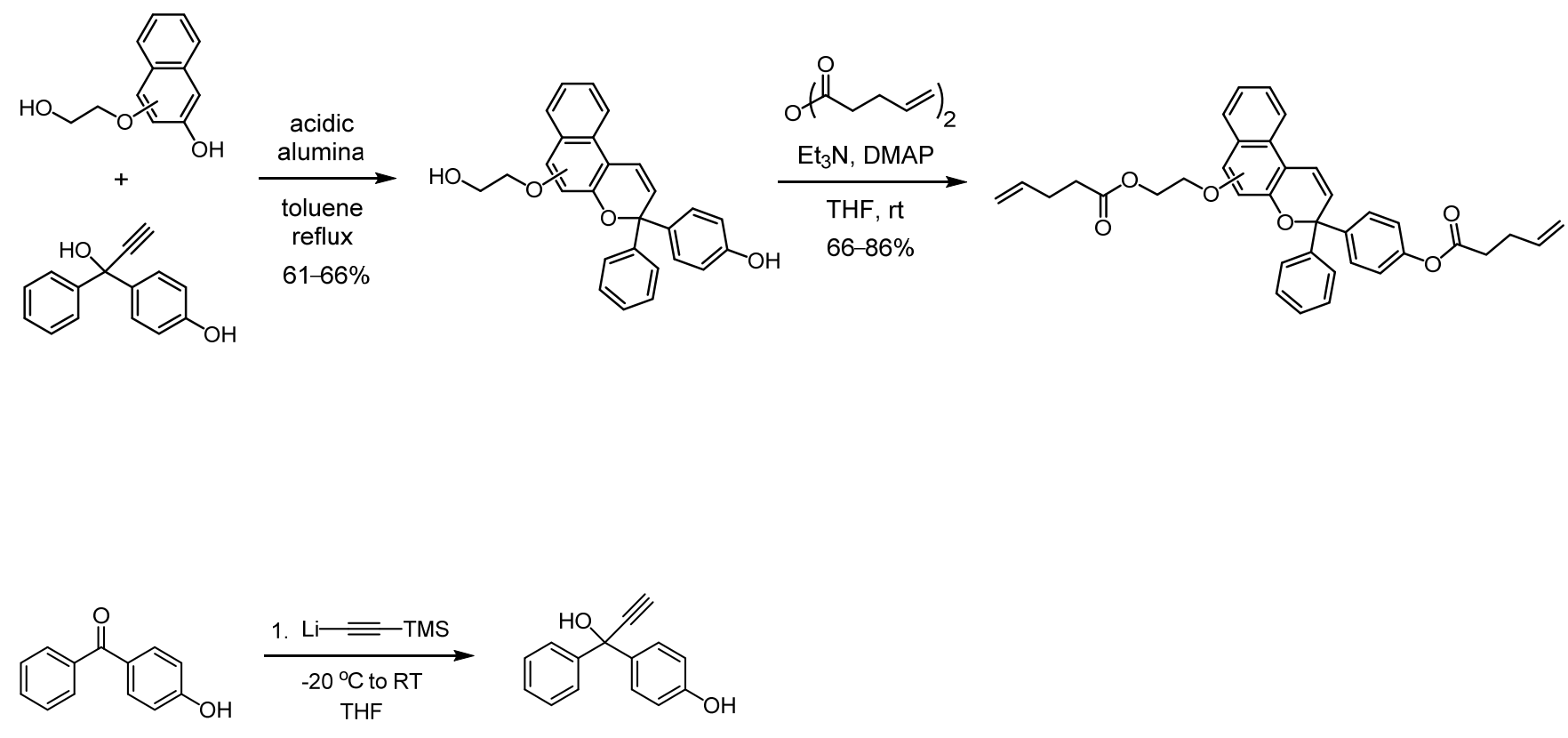

2. $\mathrm{KOH}, \mathrm{MeOH}$

1-(4-hydroxyphenyl)-1-phenylprop-2-yn-1-ol. An oven dried round bottom flask equipped with a stir bar and rubber septum was charged with ethynyltrimethylsilane $(7.60 \mathrm{~mL}, 53.8 \mathrm{mmol})$ and $150 \mathrm{~mL}$ of dry THF. The flask was cooled in a bath of ice and salt followed by the slow addition of $\mathrm{n}$-butyllithium (2.7 M in heptane, $19.8 \mathrm{~mL}, 53.5 \mathrm{mmol})$. After $1 \mathrm{~h}$, 4-hydroxybenzophenone (4.61 g, $23.3 \mathrm{mmol}$ ) dissolved separately in $10 \mathrm{~mL}$ of dry THF was added via syringe to the cold lithium acetylide mixture. After complete addition, the reaction mixture was allowed to warm to room temperature and stirred for $18 \mathrm{~h}$. The flask was subsequently cooled in ice, a solution of $\mathrm{KOH}(3.98 \mathrm{~g}, 70.9 \mathrm{mmol})$ in $15 \mathrm{~mL}$ of methanol was added via syringe, and the mixture was warmed to room temperature and stirred for $20 \mathrm{~min}$. After complete removal of the trimethylsilyl protecting group, the mixture was cooled in an ice bath and neutralized by the slow addition of acetic acid $(7.2 \mathrm{~mL})$. THF was removed under reduced pressure and the crude material was partitioned between water and ethyl acetate. The aqueous layer was discarded 
and the organic fraction was washed with $10 \% \mathrm{NaHSO}_{4}(50 \mathrm{~mL})$ and brine $(50 \mathrm{~mL})$, dried over $\mathrm{MgSO}_{4}$, filtered, and concentrated under reduced pressure. Recrystallization from toluene/hexanes (50/50) provided the title compound as a light-brown crystalline solid $(4.58 \mathrm{~g}, 88 \%) .{ }^{1} \mathrm{H} \mathrm{NMR}(500 \mathrm{MHz}$, Acetone- $\left.d_{6}\right) \delta: 3.31(\mathrm{~s}, 1 \mathrm{H}), 5.49(\mathrm{~s}, 1 \mathrm{H}), 6.74-6.79(\mathrm{~m}, 2 \mathrm{H}), 7.19-7.25(\mathrm{~m}, 1 \mathrm{H}), 7.27-7.33(\mathrm{~m}, 2 \mathrm{H}), 7.40-$ $7.45(\mathrm{~m}, 2 \mathrm{H}), 7.57-7.63(\mathrm{~m}, 2 \mathrm{H}), 8.32(\mathrm{~s}, 1 \mathrm{H}) \mathrm{ppm} .{ }^{13} \mathrm{C}\left\{{ }^{1} \mathrm{H}\right\}$ NMR (125 MHz, Acetone- $\left.d_{6}\right)$ ס: 73.8, 75.6, 88.2, 115.2, 126.5, 127.7, 128.0, 128.5, 137.7, 147.1, 157.3 ppm. HRMS (ESI, $m / z)$ : calcd for $\left[\mathrm{C}_{15} \mathrm{H}_{11} \mathrm{O}\right]^{+}$ $(\mathrm{M}-\mathrm{OH})^{+}, 207.0804$; found, 207.0802 .

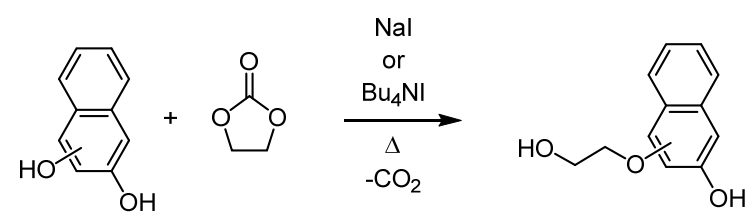

General procedure for alkylation of naphthalene diols. Hydroxyethoxy-2-naphthols were prepared via a statistical reaction of naphthalene diol (1 equiv) with ethylene carbonate (1 equiv). The reactants were combined with either sodium iodide ( $3 \mathrm{~mol} \%$ ) or tetrabutylammonium iodide $(8 \mathrm{~mol} \%)$ catalyst in a round bottom flask equipped with a stir bar and fitted with a gas bubbler. The flask was placed in a silicone oil bath maintained at $170-175{ }^{\circ} \mathrm{C}$ and reaction progress was monitored by evolution of $\mathrm{CO}_{2}$, requiring $1-3 \mathrm{~h}$ to reach completion.

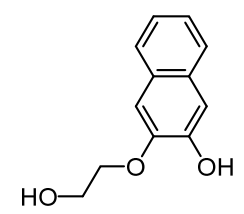

3-(2-hydroxyethoxy)naphthalene-2-ol. Following the general procedure, 2,3-dihydroxynaphthalene $(4.62 \mathrm{~g}, 28.8 \mathrm{mmol})$, ethylene carbonate $(2.55 \mathrm{~g}, 29.0 \mathrm{mmol})$, and sodium iodide (129 $\mathrm{mg}, 0.861 \mathrm{mmol})$ were heated for $2.5 \mathrm{~h}$. The resulting solid was suspended in cold dichloromethane and collected by filtration. Recrystallization from toluene afforded the title compound as a white crystalline powder (2.45 g, 42\%). ${ }^{1} \mathrm{H}$ NMR (500 MHz, DMSO-d $\left.d_{6}\right) \delta: 3.81(\mathrm{t}, J=4.9 \mathrm{~Hz}, 2 \mathrm{H}), 4.10(\mathrm{t}, J=4.9 \mathrm{~Hz}, 2 \mathrm{H}), 4.96$ (bs, $1 \mathrm{H}), 7.16(\mathrm{~s}, 1 \mathrm{H}), 7.21-7.28(\mathrm{~m}, 3 \mathrm{H}), 7.59-7.64(\mathrm{~m}, 1 \mathrm{H}), 7.65-7.70(\mathrm{~m}, 1 \mathrm{H}), 9.17$ (bs, $1 \mathrm{H}) \mathrm{ppm} .{ }^{13} \mathrm{C}\left\{{ }^{1} \mathrm{H}\right\}$ NMR (125 MHz, DMSO-d $\left.)_{6}\right)$ 8: 59.6, 70.0, 107.2, 109.5, 123.1, 123.8, 125.6, 126.3, 128.4, 129.3, 147.1, 148.0 ppm. HRMS (ESI, $m / z)$ : calcd for $\left[\mathrm{C}_{12} \mathrm{H}_{13} \mathrm{O}_{3}\right]^{+}(\mathrm{M}+\mathrm{H})^{+}, 205.0859$; found, 205.0863. 


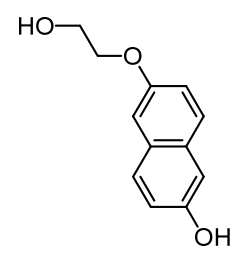

6-(2-hydroxyethoxy)naphthalene-2-ol. Following the general procedure, 2,6-dihydroxynaphthalene (1.50 g, $9.37 \mathrm{mmol})$, ethylene carbonate $(825 \mathrm{mg}, 9.37 \mathrm{mmol})$, and sodium iodide $(49.5 \mathrm{mg}, 0.330 \mathrm{mmol})$ were heated for $1 \mathrm{~h}$. The crude material was dissolved in ethyl acetate and eluted through a short plug of silica gel. The filtrate was concentrated under reduced pressure and the crude product was purified by column chromatography (7-60\% EtOAC/hexanes), followed by recrystallization from toluene to provide the title compound as a white crystalline powder (451 mg, 24\%). ${ }^{1} \mathrm{H}$ NMR (500 MHz, DMSO- $d_{6}$ ) $\delta: 3.77(\mathrm{t}, J=4.9 \mathrm{~Hz}, 2 \mathrm{H}), 4.04(\mathrm{t}, J=5.0 \mathrm{~Hz}, 2 \mathrm{H}), 4.92(\mathrm{bs}, 1 \mathrm{H}), 7.02-7.10(\mathrm{~m}, 3 \mathrm{H}), 7.19(\mathrm{~d}, J=2.5 \mathrm{~Hz}, 1 \mathrm{H})$, $7.60(\mathrm{~d}, J=8.9 \mathrm{~Hz}, 1 \mathrm{H}), 7.64(\mathrm{~d}, J=8.7 \mathrm{~Hz}, 1 \mathrm{H}), 9.46(\mathrm{bs}, 1 \mathrm{H}) \mathrm{ppm} .{ }^{13} \mathrm{C}\left\{{ }^{1} \mathrm{H}\right\} \mathrm{NMR}\left(125 \mathrm{MHz}, \mathrm{DMSO}-d_{6}\right) \delta$ : 59.7, 69.5, 106.9, 108.9, 118.8, 118.9, 127.5, 128.1, 128.6, 129.8, 153.6, 154.4 ppm. HRMS (ESI, $m / z)$ : calcd for $\left[\mathrm{C}_{12} \mathrm{H}_{13} \mathrm{O}_{3}\right]^{+}(\mathrm{M}+\mathrm{H})^{+}, 205.0859$; found, 205.0860.

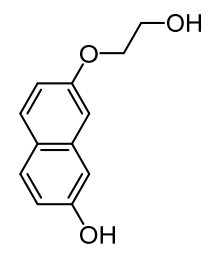

7-(2-hydroxyethoxy)naphthalene-2-ol. Following the general procedure, 2,7-dihydroxynaphthalene $(2.34 \mathrm{~g}, 14.6 \mathrm{mmol})$, ethylene carbonate $(1.29 \mathrm{~g}, 14.7 \mathrm{mmol})$, and tetrabutylammonium iodide (433 $\mathrm{mg}$, $1.17 \mathrm{mmol}$ ) were heated for $1 \mathrm{~h}$. The crude material was dissolved in ethyl acetate and eluted through a short plug of silica gel. The filtrate was concentrated under reduced pressure and the crude product was purified by column chromatography (10-80\% EtOAC/hexanes), followed by recrystallization from toluene to provide the title compound as beige needles $(1.17 \mathrm{~g}, 39 \%) .{ }^{1} \mathrm{H}$ NMR $\left(500 \mathrm{MHz}\right.$, Acetone- $\left.d_{6}\right) \delta$ : $3.92(\mathrm{q}, J=4.9 \mathrm{~Hz}, 2 \mathrm{H}), 4.04(\mathrm{bt}, J=6.0 \mathrm{~Hz}, 1 \mathrm{H}), 4.14(\mathrm{t}, J=4.9 \mathrm{~Hz}, 2 \mathrm{H}), 6.93(\mathrm{dd}, J=8.9,2.5 \mathrm{~Hz}, 1 \mathrm{H}), 6.96$ (dd, $J=8.8,2.4 \mathrm{~Hz}, 1 \mathrm{H}$ ), 7.08 (d, $J=2.5 \mathrm{~Hz}, 1 \mathrm{H}), 7.11(\mathrm{~d}, J=2.4 \mathrm{~Hz}, 1 \mathrm{H}$ ), 7.66 (d, $J=8.8 \mathrm{~Hz}, 2 \mathrm{H}), 8.59$ (bs, 1H) ppm. ${ }^{13} \mathrm{C}\left\{{ }^{1} \mathrm{H}\right\}$ NMR $\left(125 \mathrm{MHz}\right.$, Acetone- $\left.d_{6}\right) \delta: 61.4,70.5,106.2,109.2,116.6,116.6,124.8,129.9$, 130.0, 137.4, 156.7, 158.5 ppm. HRMS (ESI, $m / z)$ : calcd for $\left[\mathrm{C}_{12} \mathrm{H}_{13} \mathrm{O}_{3}\right]^{+}(\mathrm{M}+\mathrm{H})^{+}, 205.0859$; found, 205.0863. 

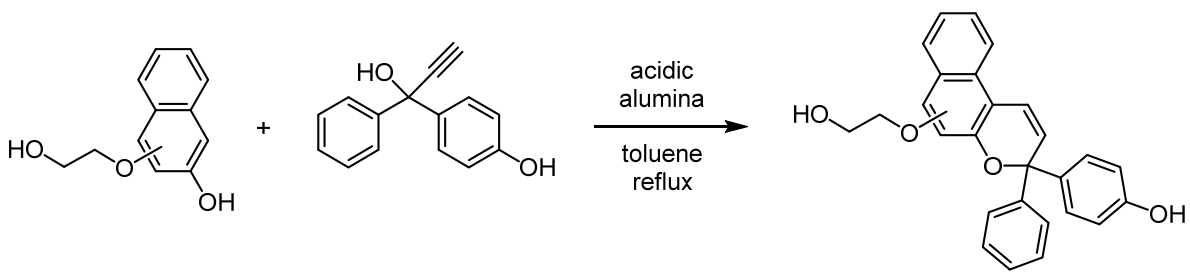

General procedure for the synthesis of naphthopyrans. Naphthopyrans were synthesized following a similar procedure by Heron and coworkers. ${ }^{1,2}$ A round bottom flask was charged with hydroxyethoxy-2naphthol (1 equiv), 1-(4-hydroxyphenyl)-1-phenylprop-2-yn-1-ol (1.3 equiv), acidic alumina, and toluene. After refluxing for 18-19 h, the reaction mixture was filtered through a short plug of silica gel, eluting with ethyl acetate. The filtrate was concentrated under reduced pressure and the crude product was purified by column chromatography.

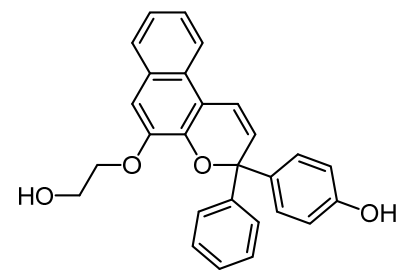

5-(2-hydroxyethoxy)-3-(4-hydroxyphenyl)-3-phenyl-3H-naphtho[2,1-b]pyran. Following the general procedure, 3-(2-hydroxyethoxy)naphthalene-2-ol $(516 \mathrm{mg}, 2.53 \mathrm{mmol}$ ) was combined with 1-(4hydroxyphenyl)-1-phenylprop-2-yn-1-ol $(739 \mathrm{mg}, 3.30 \mathrm{mmol})$ and acidic alumina $(1.98 \mathrm{~g})$ in $30 \mathrm{~mL}$ of toluene and refluxed for $18 \mathrm{~h}$. Purification by column chromatography (15-100\% EtOAC/hexanes) provided the title compound as a red solid $(683 \mathrm{mg}, 66 \%) .{ }^{1} \mathrm{H} \mathrm{NMR}\left(500 \mathrm{MHz}\right.$, Acetone- $\left.d_{6}\right) \delta: 4.00(\mathrm{t}, J=$ $5.0 \mathrm{~Hz}, 2 \mathrm{H}), 4.05(\mathrm{bs}, 1 \mathrm{H}), 4.26(\mathrm{t}, J=4.9 \mathrm{~Hz}, 2 \mathrm{H}), 6.53(\mathrm{~d}, J=9.9 \mathrm{~Hz}, 1 \mathrm{H}), 6.75-6.81(\mathrm{~m}, 2 \mathrm{H}), 7.19-7.25$ (m, 1H), 7.26-7.36 (m, 5H), 7.37-7.44 (m, 3H), 7.57-7.63 (m, 2H), 7.68 (dd, J = 7.9, 1.5 Hz, 1H), 7.96 (dd, $J=8.4,1.4 \mathrm{~Hz}, 1 \mathrm{H}), 8.37$ (bs, $1 \mathrm{H} \mathrm{ppm}) .{ }^{13} \mathrm{C}\left\{{ }^{1} \mathrm{H}\right\} \mathrm{NMR}\left(125 \mathrm{MHz}\right.$, Acetone- $\left.d_{6}\right) \delta: 61.3,71.5,83.0,110.1$, $115.5,116.6$, 120.3, 122.0, 125.0, 125.2, 126.0, 127.4, 128.0, 128.8, 129.1, 130.3, 130.6, 136.8, 143.5, 146.3, 149.3, 157.7 ppm (two peaks overlap). HRMS (ESI, $\mathrm{m} / \mathrm{z}$ ): calcd for $\left[\mathrm{C}_{27} \mathrm{H}_{23} \mathrm{O}_{4}\right]^{+}(\mathrm{M}+\mathrm{H})^{+}, 411.1591$; found, 411.1597. 


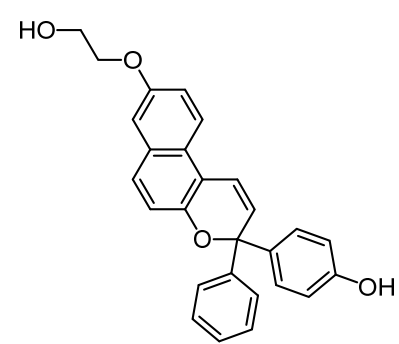

8-(2-hydroxyethoxy)-3-(4-hydroxyphenyl)-3-phenyl-3H-naphtho[2,1-b]pyran. Following the general procedure, 6-(2-hydroxyethoxy)naphthalene-2-ol $(271 \mathrm{mg}, 1.33 \mathrm{mmol})$ was combined with 1-(4hydroxyphenyl)-1-phenylprop-2-yn-1-ol $(383 \mathrm{mg}, 1.71 \mathrm{mmol})$ and acidic alumina $(1.05 \mathrm{~g})$ in $17 \mathrm{~mL}$ of toluene and refluxed for $18 \mathrm{~h}$. Purification by column chromatography (15-100\% EtOAC/hexanes) provided the title compound as a red solid $(332 \mathrm{mg}, 61 \%) .{ }^{1} \mathrm{H}$ NMR $\left(500 \mathrm{MHz}\right.$, Acetone- $\left.d_{6}\right) \delta: 3.91(\mathrm{t}, J=$ $4.8 \mathrm{~Hz}, 2 \mathrm{H}), 4.05(\mathrm{bs}, 1 \mathrm{H}), 4.13(\mathrm{t}, J=4.9 \mathrm{~Hz}, 2 \mathrm{H}), 6.40(\mathrm{~d}, J=9.9 \mathrm{~Hz}, 1 \mathrm{H}), 6.76-6.82(\mathrm{~m}, 2 \mathrm{H}), 7.13-7.26$ $(\mathrm{m}, 4 \mathrm{H}), 7.29-7.35(\mathrm{~m}, 4 \mathrm{H}), 7.39(\mathrm{~d}, J=9.8 \mathrm{~Hz}, 1 \mathrm{H}), 7.49-7.54(\mathrm{~m}, 2 \mathrm{H}), 7.63(\mathrm{~d}, J=8.8 \mathrm{~Hz}, 1 \mathrm{H}), 7.98(\mathrm{~d}, J=$ $9.2 \mathrm{~Hz}, 1 \mathrm{H}), 8.40$ (bs, $1 \mathrm{H}) \mathrm{ppm} .{ }^{13} \mathrm{C}\left\{{ }^{1} \mathrm{H}\right\}$ NMR (125 MHz, Acetone- $\left.d_{6}\right) \delta: 61.3,70.5,82.8,108.5,115.4$, 115.6, 119.4, 120.2, 120.3, 123.8, 125.9, 127.5, 128.0, 128.8, 129.2, 129.3, 129.7, 131.4, 136.9, 146.5, 149.8, 156.4, 157.6 ppm. HRMS $(E S I, m / z)$ : calcd for $\left[\mathrm{C}_{27} \mathrm{H}_{23} \mathrm{O}_{4}\right]^{+}(\mathrm{M}+\mathrm{H})^{+}, 411.1591$; found, 411.1591 .

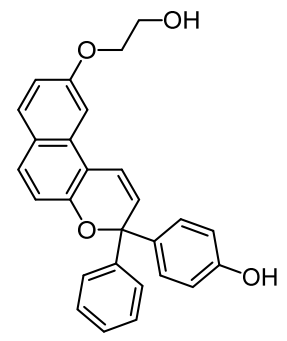

9-(2-hydroxyethoxy)-3-(4-hydroxyphenyl)-3-phenyl-3H-naphtho[2,1-b]pyran. Following the general procedure, 7-(2-hydroxyethoxy)naphthalene-2-ol (394 mg, $1.93 \mathrm{mmol}$ ) was combined with 1-(4hydroxyphenyl)-1-phenylprop-2-yn-1-ol $(567 \mathrm{mg}, 2.53 \mathrm{mmol})$ and acidic alumina $(1.52 \mathrm{~g})$ in $24 \mathrm{~mL}$ of toluene and refluxed for $19 \mathrm{~h}$. Purification by column chromatography (15-100\% EtOAC/hexanes) provided the title compound as a red solid (496 mg, 63\%). $\left.{ }^{1} \mathrm{H} \mathrm{NMR} \mathrm{(500} \mathrm{MHz,} \mathrm{Acetone-} d_{6}\right) \delta: 3.96(\mathrm{t}, J=$ $4.9 \mathrm{~Hz}, 2 \mathrm{H}), 4.24(\mathrm{t}, J=4.9 \mathrm{~Hz}, 2 \mathrm{H}), 6.37(\mathrm{~d}, J=10.0 \mathrm{~Hz}, 1 \mathrm{H}), 6.80-6.85(\mathrm{~m}, 2 \mathrm{H}), 7.00(\mathrm{dd}, J=8.9,2.4 \mathrm{~Hz}$, 1H), $7.07(\mathrm{~d}, J=8.7 \mathrm{~Hz}, 1 \mathrm{H}), 7.21-7.26(\mathrm{~m}, 1 \mathrm{H}), 7.29-7.37(\mathrm{~m}, 4 \mathrm{H}), 7.42(\mathrm{~d}, J=10.1 \mathrm{~Hz}, 1 \mathrm{H}), 7.44(\mathrm{~d}, J=$ $2.4 \mathrm{~Hz}, 1 \mathrm{H}), 7.50-7.55(\mathrm{~m}, 2 \mathrm{H}), 7.65$ (dd, $J=10.1,8.9 \mathrm{~Hz}, 2 \mathrm{H}), 8.45$ (bs, $1 \mathrm{H}) \mathrm{ppm} .{ }^{13} \mathrm{C}\left\{{ }^{1} \mathrm{H}\right\} \mathrm{NMR}(125 \mathrm{MHz}$, Acetone- $\left.d_{6}\right) \delta: 61.2,70.3,82.7,102.0,114.2,115.4,116.2,116.9,120.1,125.4,127.2,127.8,128.5$, 
128.6, 129.0, 130.2, 130.6, 132.0, 136.7, 146.3, 151.8, 157.4, $158.8 \mathrm{ppm}$. HRMS (ESI, $\mathrm{m} / \mathrm{z}$ ): calcd for $\left[\mathrm{C}_{27} \mathrm{H}_{23} \mathrm{O}_{4}\right]^{+}(\mathrm{M}+\mathrm{H})^{+}, 411.1591$; found, 411.1599 .

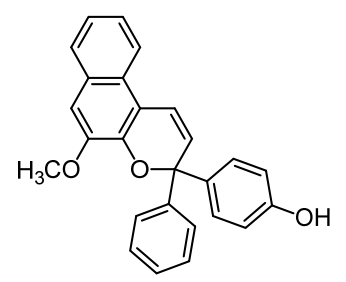

5-methoxy-3-(4-hydroxyphenyl)-3-phenyl-3H-naphtho[2,1-b]pyran. Following the general procedure, 3-methoxynaphthalene-2-ol ${ }^{3}$ (332 mg, $1.91 \mathrm{mmol}$ ) was combined with 1-(4-hydroxyphenyl)-1phenylprop-2-yn-1-ol (564 mg, $2.52 \mathrm{mmol}$ ) and acidic alumina (1.49 g) in $22 \mathrm{~mL}$ of toluene and refluxed for $18 \mathrm{~h}$. Purification by column chromatography (10-70\% EtOAC/hexanes) provided the title compound as a red solid (408 mg, 56\%). ${ }^{1} \mathrm{H}$ NMR (500 MHz, Acetone- $\left.d_{6}\right) \delta: 3.97(\mathrm{~s}, 3 \mathrm{H}), 6.51(\mathrm{~d}, J=9.9 \mathrm{~Hz}, 1 \mathrm{H})$, 6.78-6.83 (m, 2H), 7.21-7.25 (m, 1H), 7.26-7.35 (m, 5H), 7.36-7.43 (m, 3H), 7.54-7.60 (m, 2H), 7.67$7.73(\mathrm{~m}, 1 \mathrm{H}), 7.96(\mathrm{dd}, J=8.1,1.4 \mathrm{~Hz}, 1 \mathrm{H}), 8.46$ (bs, $1 \mathrm{H}) \mathrm{ppm} .{ }^{13} \mathrm{C}\left\{{ }^{1} \mathrm{H}\right\}$ NMR $\left(125 \mathrm{MHz}\right.$, Acetone- $\left.d_{6}\right) \delta$ : 56.1, 83.0, 108.5, 115.5, 116.1, 120.2, 122.0, 125.0, 125.1, 125.8, 127.3, 128.0, 128.0, 128.7, 129.0, $129.9,130.5,136.7,143.1,146.2,149.9,157.7 \mathrm{ppm}$. HRMS (ESI, $\mathrm{m} / \mathrm{z})$ : calcd for $\left[\mathrm{C}_{26} \mathrm{H}_{21} \mathrm{O}_{3}\right]^{+}(\mathrm{M}+\mathrm{H})^{+}$, 381.1485; found, 381.1476.
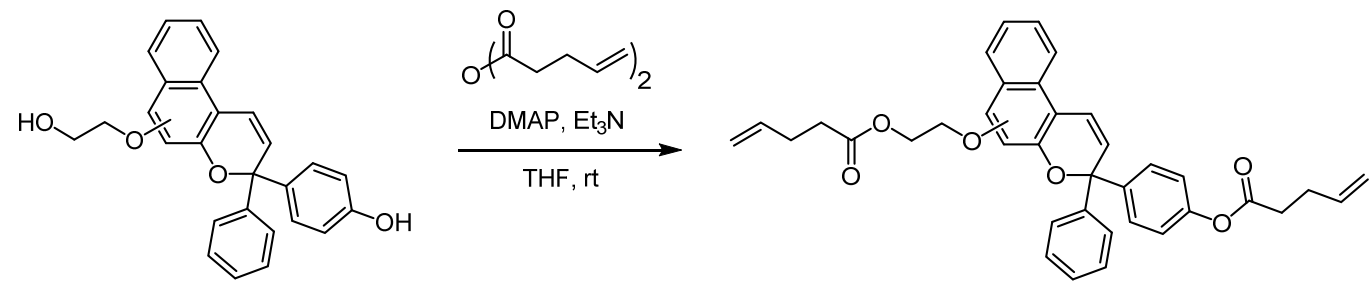

General procedure for functionalization of naphthopyrans. Naphthopyrans were functionalized with terminal alkene groups to facilitate covalent incorporation into PDMS networks via Pt-catalyzed hydrosilylation chemistry. ${ }^{4}$ The hydroxyl-functionalized naphthopyran was combined with 4-pentenoic anhydride, triethylamine, and DMAP in THF and stirred overnight at room temperature. The reaction mixture was diluted with ethyl acetate and washed consecutively with $10 \% \mathrm{NaHSO}_{4}(2 \times 50 \mathrm{~mL}), 10 \%$ $\mathrm{NaHCO}_{3}(2 \times 50 \mathrm{~mL})$, and brine $(50 \mathrm{~mL})$. The organic phase was dried over $\mathrm{MgSO}_{4}$, filtered, concentrated under reduced pressure, and the crude product was purified by column chromatography. 


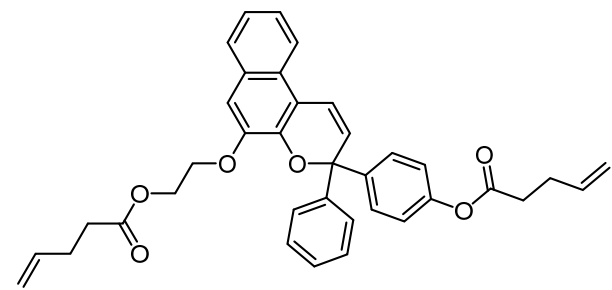

5-(2-(pent-4-enoyloxy)ethoxy)-3-(4-(pent-4-enoyloxy)phenyl)-3-phenyl-3H-naphtho[2,1-b]pyran

(NP5). Following the general procedure, 5-(2-hydroxyethoxy)-3-(4-hydroxyphenyl)-3-phenyl-3Hnaphtho[2,1-b]pyran (591 mg, $1.44 \mathrm{mmol}$ ) was combined with 4-pentenoic anhydride (605 $\mu \mathrm{L}, 3.31$ mmol), triethylamine (425 $\mu \mathrm{L}, 3.05 \mathrm{mmol})$, and DMAP $(44.0 \mathrm{mg}, 0.360 \mathrm{mmol})$ in $14 \mathrm{~mL}$ of THF and stirred overnight at room temperature. Purification by column chromatography (5-50\% EtOAC/hexanes) provided the title compound as an orange, viscous oil (708 mg, 86\%). ${ }^{1} \mathrm{H}$ NMR (500 MHz, Acetone- $\left.d_{6}\right) \delta$ : 2.31-2.38 (m, 2H), 2.39-2.49 (m, 4H), $2.64(\mathrm{t}, J=7.3 \mathrm{~Hz}, 2 \mathrm{H}), 4.40-4.45(\mathrm{~m}, 2 \mathrm{H}), 4.54-4.60(\mathrm{~m}, 2 \mathrm{H})$, 4.88-5.14 (m, 4H), 5.77-5.94 (m, 2H), $6.65(\mathrm{~d}, J=9.9 \mathrm{~Hz}, 1 \mathrm{H}), 7.05-7.10(\mathrm{~m}, 2 \mathrm{H}), 7.22-7.27(\mathrm{~m}, 1 \mathrm{H})$, 7.28-7.38 (m, 5H), $7.48(\mathrm{~d}, J=9.9 \mathrm{~Hz}, 1 \mathrm{H}), 7.61-7.67(\mathrm{~m}, 4 \mathrm{H}), 7.68-7.72(\mathrm{~m}, 1 \mathrm{H}), 7.99(\mathrm{dd}, J=8.2,1.3 \mathrm{~Hz}$, 1H) ppm. ${ }^{13} \mathrm{C}\left\{{ }^{1} \mathrm{H}\right\}$ NMR (125 MHz, Acetone- $d_{6}$ ) $\delta: 29.6,29.7,34.0,34.0$ (peaks overlap), 63.4, 67.8, 82.7, $110.4,115.9,116.1,116.8,120.9,122.2,122.3,125.3,125.5,126.1,127.3,128.2,128.4,128.5,129.0$, $129.8,130.6$, 137.7, 137.9, 143.2, 143.4, 145.8, 148.7, 151.2, 171.7, 173.1 ppm. HRMS (ESI, m/z): calcd for $\left[\mathrm{C}_{37} \mathrm{H}_{35} \mathrm{O}_{6}\right]^{+}(\mathrm{M}+\mathrm{H})^{+}$, 575.2428; found, 575.2432.

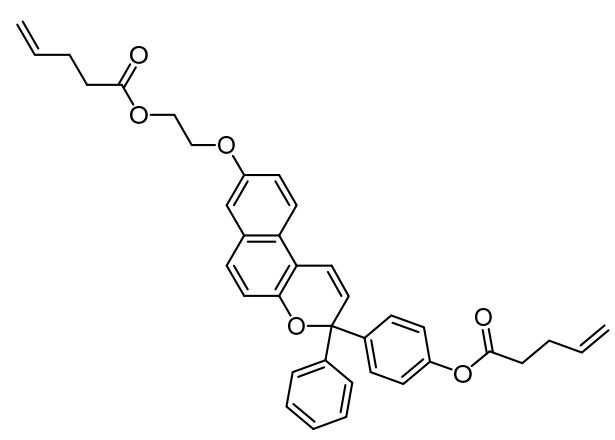

8-(2-(pent-4-enoyloxy)ethoxy)-3-(4-(pent-4-enoyloxy)phenyl)-3-phenyl-3H-naphtho[2,1-b]pyran

(NP8). Following the general procedure, 8-(2-hydroxyethoxy)-3-(4-hydroxyphenyl)-3-phenyl-3Hnaphtho[2,1-b]pyran (225 mg, $0.548 \mathrm{mmol}$ ) was combined with 4-pentenoic anhydride (220 $\mu \mathrm{L}, 1.20$ mmol), triethylamine $(170 \mu \mathrm{L}, 1.22 \mathrm{mmol})$, and DMAP $(15.9 \mathrm{mg}, 0.130 \mathrm{mmol})$ in $5 \mathrm{~mL}$ of THF and stirred overnight at room temperature. Purification by column chromatography (5-40\% EtOAC/hexanes) provided the title compound as a yellow, viscous oil (208 mg, 66\%). ${ }^{1} \mathrm{H}$ NMR (500 MHz, Acetone- $\left.d_{6}\right) \delta$ : 
2.30-2.37 (m, 2H), 2.39-2.46 (m, 4H), $2.65(\mathrm{t}, J=7.3 \mathrm{~Hz}, 2 \mathrm{H}), 4.27-4.31(\mathrm{~m}, 2 \mathrm{H}), 4.43-4.47(\mathrm{~m}, 2 \mathrm{H})$, 4.91-5.14 (m, 4H), 5.78-5.95 (m, 2H), $6.48(\mathrm{~d}, J=9.9 \mathrm{~Hz}, 1 \mathrm{H}), 7.06-7.11(\mathrm{~m}, 2 \mathrm{H}), 7.18(\mathrm{dd}, J=9.2,2.6 \mathrm{~Hz}$, 1H), 7.21-7.28 (m, 3H), 7.31-7.36 (m, 2H), $7.45(\mathrm{~d}, J=10.0 \mathrm{~Hz}, 1 \mathrm{H}), 7.52-7.57(\mathrm{~m}, 4 \mathrm{H}), 7.66(\mathrm{~d}, J=8.8 \mathrm{~Hz}$, 1H), $8.01(\mathrm{~d}, J=9.2 \mathrm{~Hz}, 1 \mathrm{H}) \mathrm{ppm} .{ }^{13} \mathrm{C}\left\{{ }^{1} \mathrm{H}\right\} \mathrm{NMR}\left(125 \mathrm{MHz}\right.$, Acetone- $\left.d_{6}\right) \delta: 29.6,29.7,33.9,34.0,63.5$, 67.0, 82.7, 108.8, 115.5, 115.8, 116.1, 119.6, 120.3, 120.8, 122.3, 124.1, 126.1, 127.6, 128.4, 128.8, 129.1, 129.3, 129.7, 131.5, 137.7, 137.9, 143.5, 145.9, 149.8, 151.2, 156.2, 171.8, 173.1 ppm. HRMS (ESI, $m / z)$ : calcd for $\left[\mathrm{C}_{37} \mathrm{H}_{35} \mathrm{O}_{6}\right]^{+}(\mathrm{M}+\mathrm{H})^{+}, 575.2428$; found, 575.2428 .

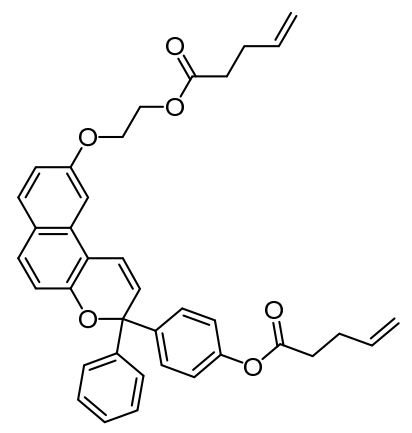

\section{9-(2-(pent-4-enoyloxy)ethoxy)-3-(4-(pent-4-enoyloxy)phenyl)-3-phenyl-3H-naphtho[2,1-b]pyran}

(NP9). Following the general procedure, 9-(2-hydroxyethoxy)-3-(4-hydroxyphenyl)-3-phenyl-3Hnaphtho[2,1-b]pyran (276 mg, $0.672 \mathrm{mmol}$ ) was combined with 4-pentenoic anhydride $(270 \mu \mathrm{L}, 1.48$ mmol), triethylamine $(210 \mu \mathrm{L}, 1.51 \mathrm{mmol})$, and DMAP $(17.1 \mathrm{mg}, 0.140 \mathrm{mmol})$ in $6 \mathrm{~mL}$ of THF and stirred overnight at room temperature. Purification by column chromatography ( $5-40 \%$ EtOAC/hexanes) provided the title compound as a yellow, viscous oil (316 mg, 82\%). ${ }^{1} \mathrm{H}$ NMR (500 MHz, Acetone- $\left.d_{6}\right) \delta$ : 2.31-2.37 (m, 2H), 2.39-2.47 (m, 4H), $2.65(\mathrm{t}, J=7.4 \mathrm{~Hz}, 2 \mathrm{H}), 4.37-4.42(\mathrm{~m}, 2 \mathrm{H}), 4.45-4.50(\mathrm{~m}, 2 \mathrm{H})$, 4.92-5.14 (m, 4H), 5.79-5.95 (m, 2H), $6.46(\mathrm{~d}, J=10.0 \mathrm{~Hz}, 1 \mathrm{H}), 7.02(\mathrm{dd}, J=8.9,2.4 \mathrm{~Hz}, 1 \mathrm{H}), 7.07-7.13$ (m, 3H), 7.23-7.28 (m, 1H), 7.31-7.37 (m, 2H), $7.48(\mathrm{~d}, J=2.5 \mathrm{~Hz}, 1 \mathrm{H}), 7.50(\mathrm{~d}, J=10.0 \mathrm{~Hz}, 1 \mathrm{H}), 7.52-7.58$

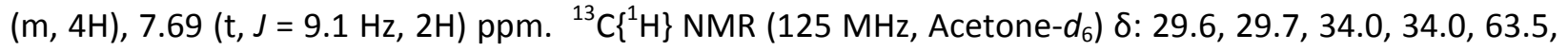
67.0, 82.8, 102.5, 114.6, 115.9, 116.1, 116.7, 117.2, 120.9, 122.3, 126.0, 127.6, 128.4, 128.4, 128.8, $129.1,130.7,131.1,132.2,137.7,137.9,143.5,145.9,151.2,152.0,158.8,171.8,173.2$ ppm. HRMS (ESI, $m / z)$ : calcd for $\left[\mathrm{C}_{37} \mathrm{H}_{35} \mathrm{O}_{6}\right]^{+}(\mathrm{M}+\mathrm{H})^{+}, 575.2428$; found, 575.2420. 


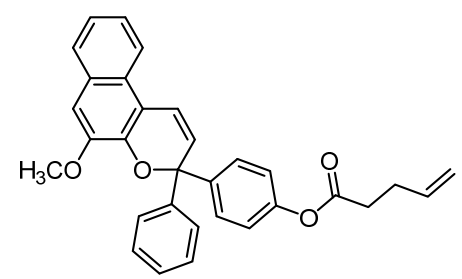

5-methoxy-3-(4-(pent-4-enoyloxy)phenyl)-3-phenyl-3H-naphtho[2,1-b]pyran (Control). Following the general procedure, 5-methoxy-3-(4-hydroxyphenyl)-3-phenyl-3H-naphtho[2,1-b]pyran (181 mg, 0.476 mmol) was combined with 4-pentenoic anhydride $(100 \mu \mathrm{L}, 0.547 \mathrm{mmol})$, triethylamine $(75 \mu \mathrm{L}, 0.54$ mmol), and DMAP (8.2 mg, $0.067 \mathrm{mmol})$ in $5 \mathrm{~mL}$ of THF and stirred overnight at room temperature. Purification by column chromatography (2-40\% EtOAC/hexanes) provided the title compound as a yellow-orange, sticky solid (197 mg, 90\%). ${ }^{1} \mathrm{H}$ NMR (500 MHz, Acetone- $\left.d_{6}\right) \delta:$ 2.39-2.45 (m, 2H), $2.64(\mathrm{t}$, $J=7.4 \mathrm{~Hz}, 2 \mathrm{H}$ ), $3.99(\mathrm{~s}, 3 \mathrm{H}), 5.01(\mathrm{dq}, J=10.2,1.4 \mathrm{~Hz}, 1 \mathrm{H}), 5.11(\mathrm{dq}, J=17.2,1.7 \mathrm{~Hz}, 1 \mathrm{H}), 5.90(\mathrm{ddt}, J=$ $16.9,10.3,6.5 \mathrm{~Hz}, 1 \mathrm{H}), 6.59$ (d, J = 9.9 Hz, 1H), 7.06-7.11 (m, 2H), 7.22-7.27 (m, 1H), 7.28-7.37 (m, 5H), $7.46(\mathrm{~d}, J=9.9 \mathrm{~Hz}, 1 \mathrm{H}), 7.57-7.62(\mathrm{~m}, 4 \mathrm{H}), 7.69-7.73(\mathrm{~m}, 1 \mathrm{H}), 7.97(\mathrm{dd}, J=8.3,1.4 \mathrm{~Hz}, 1 \mathrm{H}) \mathrm{ppm} .{ }^{13} \mathrm{C}\left\{{ }^{1} \mathrm{H}\right\}$ NMR (125 MHz, Acetone- $\left.d_{6}\right)$ ס: 29.6, 34.0, 56.3, 82.9, 108.8, 116.1, 116.4, 120.9, 122.2, 122.3, 125.3, $125.3,125.9,127.5,128.2,128.5,128.7,129.1,129.6,130.8,137.7,143.1,143.4,145.8,150.0,151.3$, 171.8 ppm. HRMS (ESI, $\mathrm{m} / z)$ : calcd for $\left[\mathrm{C}_{31} \mathrm{H}_{27} \mathrm{O}_{4}\right]^{+}(\mathrm{M}+\mathrm{H})^{+}, 463.1904$; found, 463.1895 .

\section{Hydrosilylation Control Experiments}

In order to rule out reaction of the pyran double bond under Pt catalyzed hydrosilylation conditions (i.e., polymer curing reaction), 28-29 mg of each vinyl-functionalized naphthopyran regioisomer (NP5, NP8, and NP9) were reacted with an excess of triethoxysilane (25 equiv) using Karstedt's catalyst (5 $\mu$ L, 2 wt\% Pt in xylene). After $3 \mathrm{~h}$, excess triethoxysilane was removed under vacuum and the crude reaction mixtures were analyzed by ${ }^{1} \mathrm{H}$ NMR spectroscopy (Figure S2). The data demonstrate complete conversion of the vinyl groups, while the doublet between 6.4 and $6.7 \mathrm{ppm}(\mathrm{J} \approx 10 \mathrm{~Hz})$ diagnostic of $2-\mathrm{H}$ on the pyran ring (labeled a) remains untouched. 
a

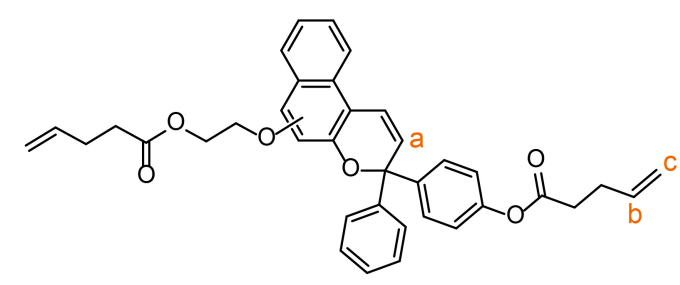

$\downarrow \begin{aligned} & \mathrm{HSi}(\mathrm{OEt}) 3 \quad \text { (25 equiv) } \\ & \text { Karstedt's } \\ & \text { catalyst }\end{aligned}$

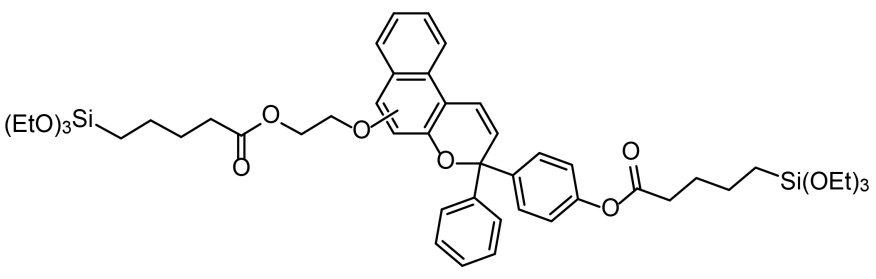

C
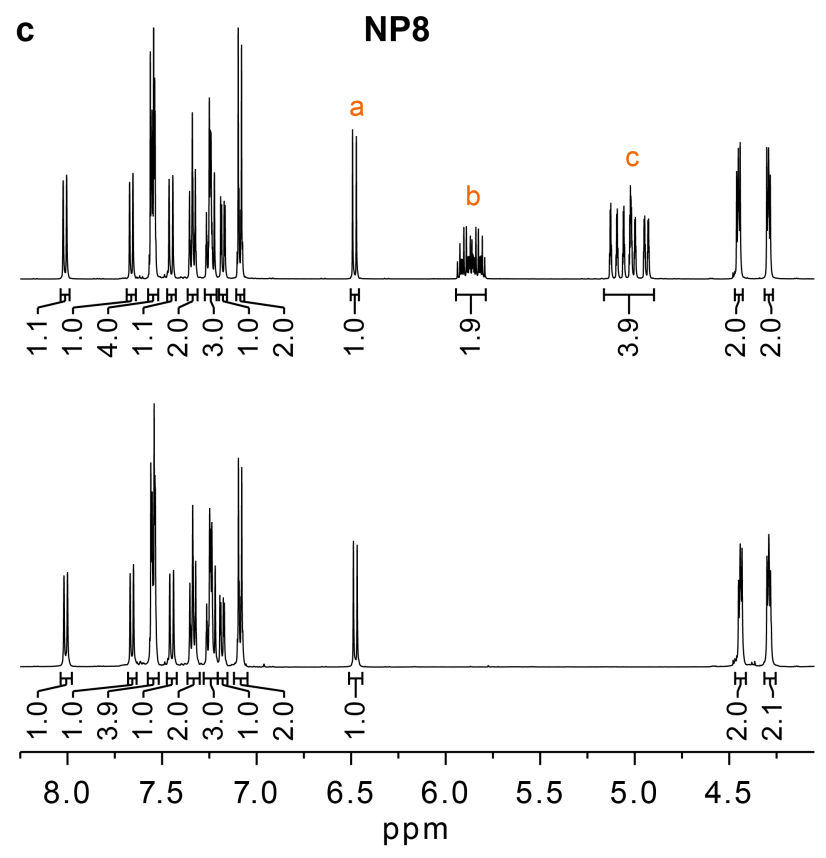

b
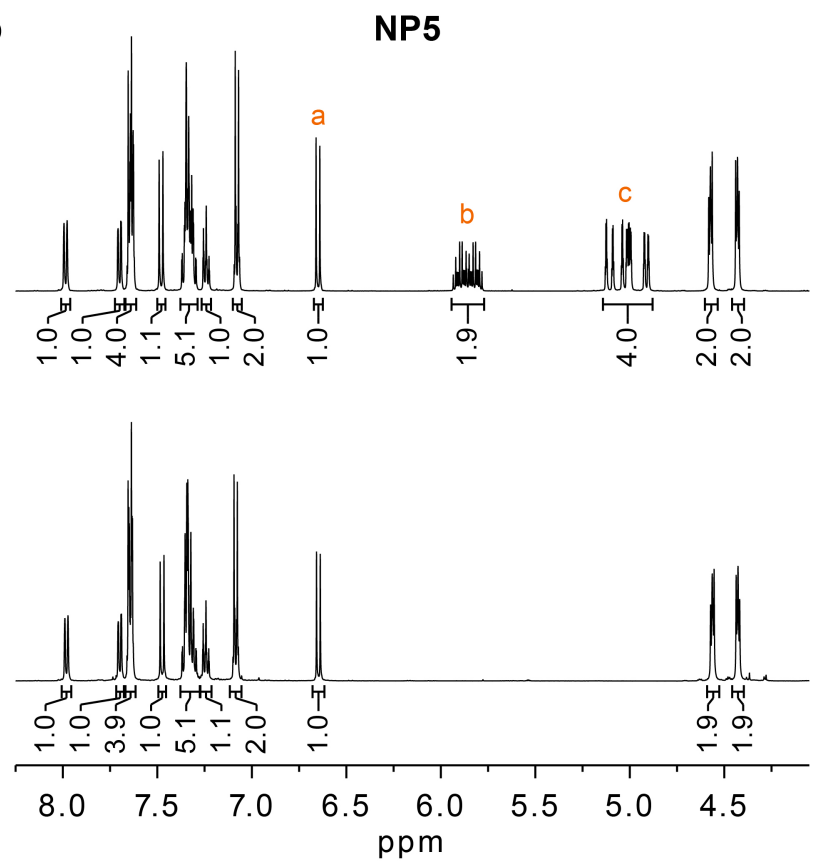

d
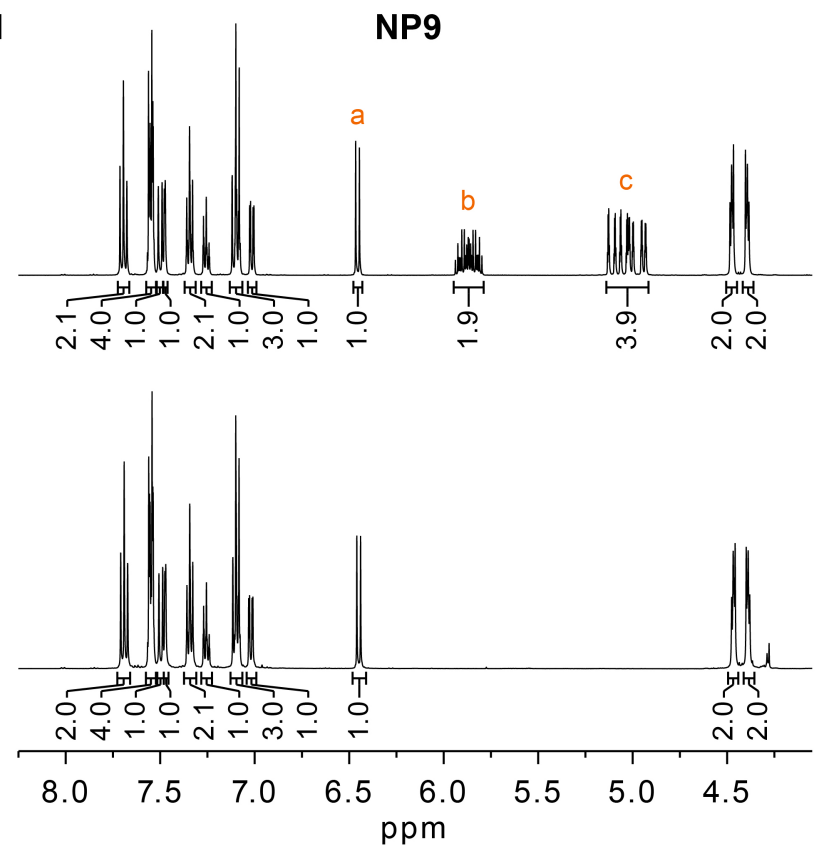

Figure S2. Results of the hydrosilylation control experiments. (a) Vinyl-functionalized naphthopyran regioisomers were reacted with 25 equiv of triethoxysilane and Karstedt's catalyst. (b-d) ${ }^{1} \mathrm{H}$ NMR spectra (acetone- $d_{6}, 500 \mathrm{MHz}$ ) before (top) and after (bottom) the reaction demonstrate selective hydrosilylation of the vinyl groups, while the pyran double bond remains untouched. 


\section{Preparation of PDMS Materials}

PDMS materials incorporating naphthopyrans (1.5 wt\%) were prepared using the two-part Sylgard ${ }^{\circledR} 184$ elastomer (Dow Corning). PDMS sheets were cut into $5 \mathrm{~mm} \times 30 \mathrm{~mm}$ strips ( $0.75 \pm 0.06 \mathrm{~mm}$ thick) for manual testing. Dogbone shaped specimens (gauge length: $5 \mathrm{~mm}$; width: $2 \mathrm{~mm}$; thickness: $0.7 \mathrm{~mm}$ ) were used for in situ visible absorption measurements during mechanical testing with a custom uniaxial load frame (see below).

General procedure for preparation of PDMS strips. A representative procedure is provided for PDMS incorporating the mechanochemically active bis-functional naphthopyran mechanophore substituted at the 5-position. 5-(2-(Pent-4-enoyloxy)ethoxy)-3-(4-(pent-4-enoyloxy)phenyl)-3-phenyl-3H-naphtho[2,1b]pyran (NP5) (31.9 mg) was dissolved in $0.1 \mathrm{~mL}$ xylene in a $20 \mathrm{~mL}$ scintillation vial. Sylgard ${ }^{\circledR} 184$ prepolymer base (1.913 g) was added and the mixture was stirred to form a homogenous, pale yellow opaque dispersion. Sylgard ${ }^{\circledR} 184$ curing agent $(190.6 \mathrm{mg}$ ) was added and the contents were mixed extensively using a vortex mixer. The mixture was pipetted onto a $5 \mathrm{~cm} \times 5 \mathrm{~cm}$ delrin plate which was placed inside a vacuum chamber and evacuated under high vacuum ( $<50 \mathrm{mTorr}$ ) for approximately $3 \mathrm{~h}$. The delrin plate was then transferred to an oven and cured at $80^{\circ} \mathrm{C}$ ( $1 \mathrm{~atm}$ pressure). After $18 \mathrm{~h}$, the plate was removed from the oven and the PDMS film was peeled off and cut into $5 \mathrm{~mm} \times 30 \mathrm{~mm}$ strips for testing. After curing, the PDMS material is clear and slightly yellow in color. PDMS materials incorporating the other naphthopyran regioisomers (and mono-functional control) were prepared in a similar fashion.

General procedure for preparation of dogbone specimens for mechanical characterization. A representative procedure is provided for PDMS incorporating the mechanochemically active bisfunctional naphthopyran mechanophore substituted at the 5-position. 5-(2-(Pent-4-enoyloxy)ethoxy)-3(4-(pent-4-enoyloxy)phenyl)-3-phenyl-3H-naphtho[2,1-b]pyran (NP5) $(17.0 \mathrm{mg}$ ) was dissolved in $0.2 \mathrm{~mL}$ xylene. Sylgard ${ }^{\circledR} 184$ prepolymer base $(1.03 \mathrm{~g})$ and curing agent $(103 \mathrm{mg})$ were added and the contents were thoroughly mixed for 10 min using a vortex mixer. The mixture was then poured into a dogboneshaped delrin mold and cured under vacuum at $65^{\circ} \mathrm{C}$ for 24 hours. 


\section{In Situ Mechanical/Visible Absorption Measurements}

Visible absorption spectra were acquired for dogbone shaped PDMS specimens using a custom uniaxial load frame (IMAC Motion Control Corp.). The testing setup is illustrated below in Figure S3. The specimens were uniaxially stretched at a strain rate of $0.01 \mathrm{~s}^{-1}$ by two opposing actuators and the load was monitored with a 50-lb capacity Honeywell Sensotec sensor. Absorption spectra were acquired using an Ocean Optics D-2000 deuterium light source and Ocean Optics HR2000+ spectrometer. A 400 $\mathrm{nm}$ longpass filter was placed between the specimen and the light source to exclude UV light. After the specimen was deformed in tension to a maximum stretch ratio of 2.8-2.9, absorption spectra were collected every $30 \mathrm{~min}$. True stress was calculated by measuring the dimensions of the deformed specimen.

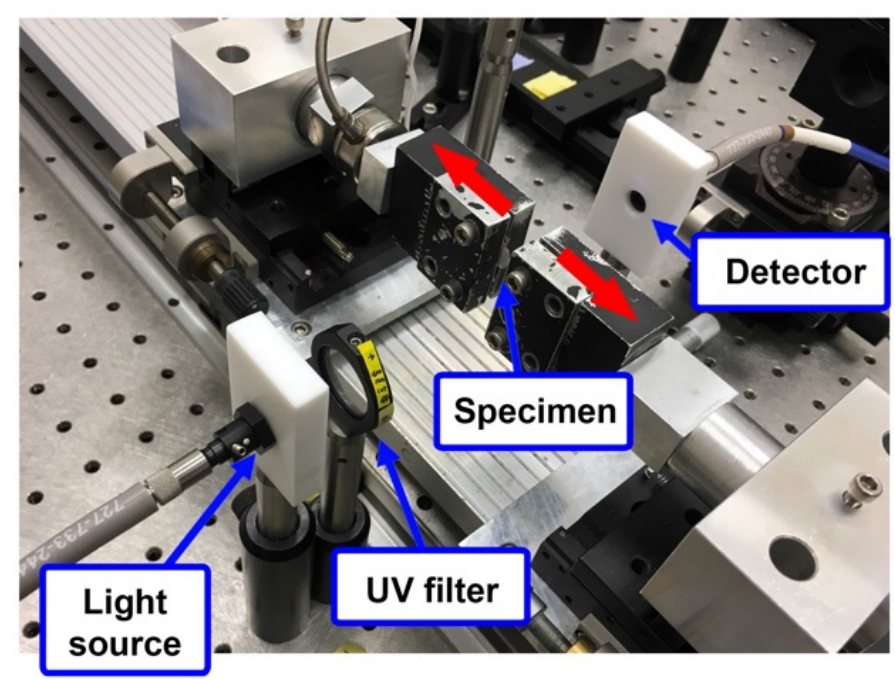

Figure S3. Mechanical testing setup with in situ visible absorption characterization.

\section{Computational Calculations}

CoGEF calculations were performed using Spartan '14 according to previously reported methods. ${ }^{5-7}$ Ground state energies were calculated using DFT at the B3LYP/6-31G* level of theory. Starting from the equilibrium geometry of the unconstrained molecules (energy $=0 \mathrm{~kJ} / \mathrm{mol}$ ), the distance between the terminal methyl ether groups of the naphthopyran structures was increased in increments of $0.1 \AA$ (0.05 $\AA$ in the region surrounding the breaking point) and the energy was minimized at each interval. Calculated structures for the three naphthopyran regioisomers are displayed below in Figure S4 at 
points of elongation corresponding to the equilibrium (i.e., force-free) geometry, and immediately before and after bond cleavage. Only the naphthopyran with substitution at the 5-position is predicted to undergo mechanical cleavage of the $\mathrm{C}-\mathrm{O}$ pyran bond with transformation into the merocyanine species. In contrast, homolytic cleavage of the terminal $\mathrm{CH}_{3}-\mathrm{O}$ bond was predicted for the naphthopyran regioisomers substituted at the 8- and 9-position. The maximum force associated with each covalent transformation was calculated from the slope of the curve immediately prior to bond cleavage.

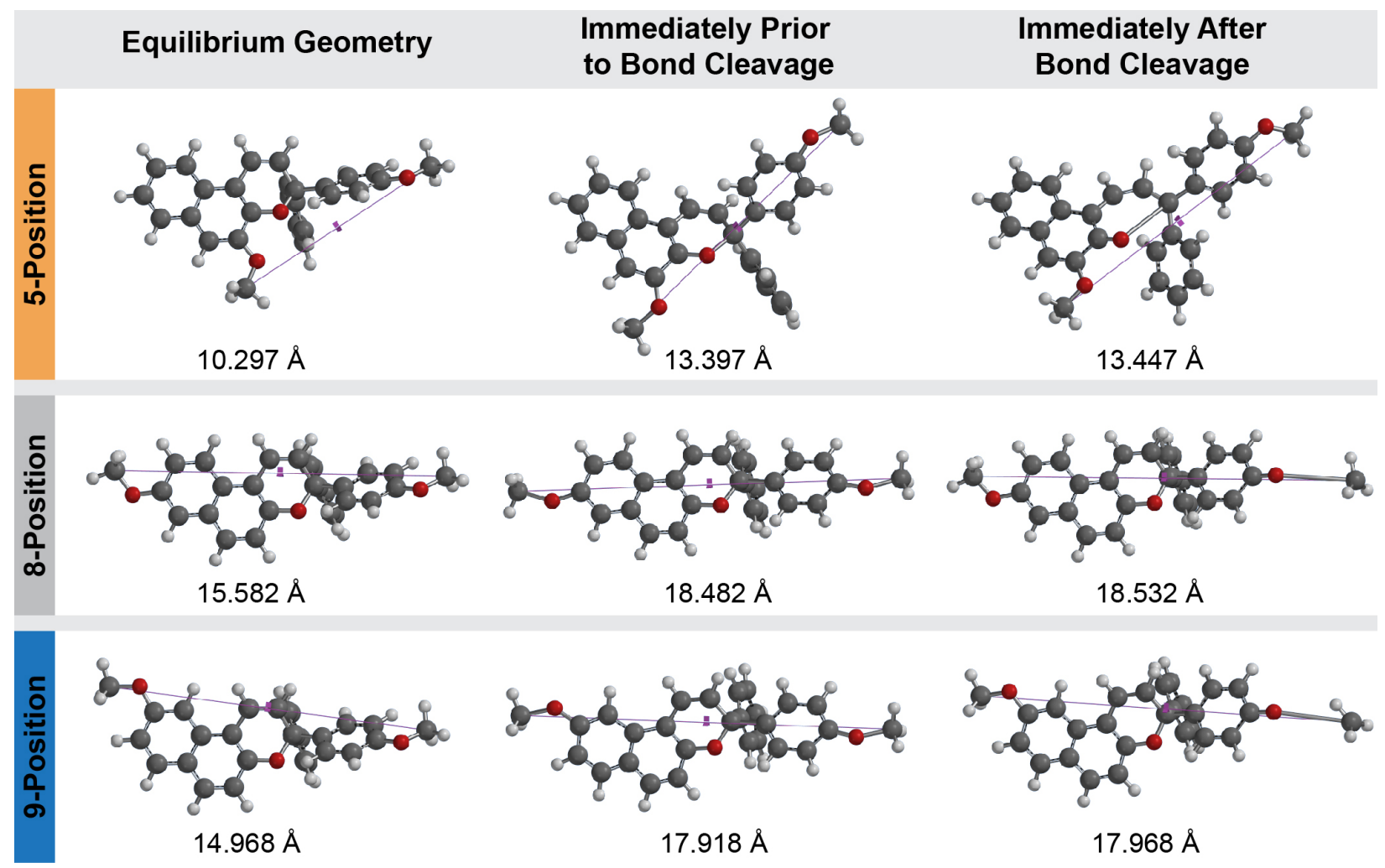

Figure S4. Structures of the three naphthopyran regioisomers at various points of elongation during the CoGEF calculations (B3LYP/6-31G*). 
The structures of naphthopyran regioisomers from CoGEF calculations taken immediately prior to bond cleavage were saved as Protein Data Bank (pdb) files and imported into Vesta. ${ }^{8}$ The atomic coordinates were then used to calculate representative vectors, as illustrated below in Figure S5. Oxygen atoms (labeled A and B) were chosen as end points to approximate the external force vector to minimize the number of degrees of freedom compared to the methyl carbon atoms, where small conformational changes result in significantly different directionality of the calculated force.

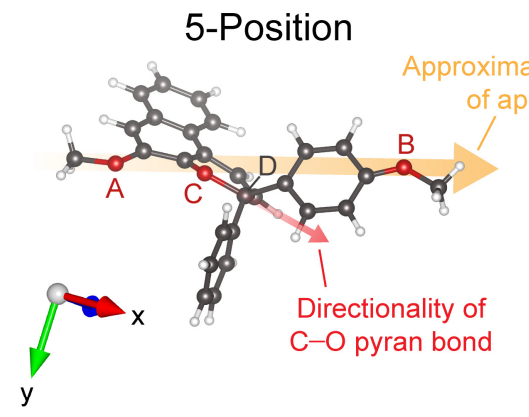

Atom (Coordinates (x,y,z))

A $(-3.104,0.819,-2.958)$

B $(5.823,-2.104,1.809)$

C $(-0.373,0.207,-1.290)$

D $(0.859,0.542,-0.209)$

$\angle$ between $\overrightarrow{\mathrm{AB}}$ and $\overrightarrow{\mathrm{CD}}=\mathbf{3 1 ^ { \circ }}$
8-Position

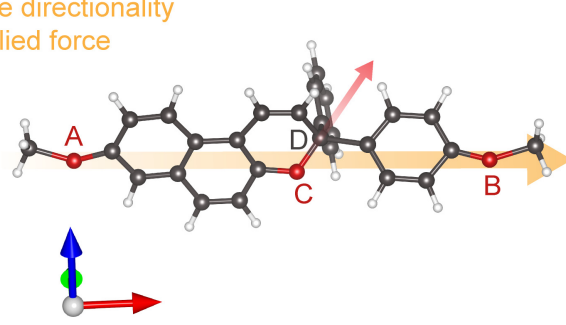

Atom (Coordinates (x,y,z))

A $(-7.767,-0.309,-0.139)$

B $(7.376,-1.407,-0.261)$

C $(0.338,-0.762,-0.745)$

D $(1.250,-0.268,0.346)$

$\angle$ between $\overrightarrow{\mathrm{AB}}$ and $\overrightarrow{\mathrm{CD}}=\mathbf{5} 5^{\circ}$
9-Position

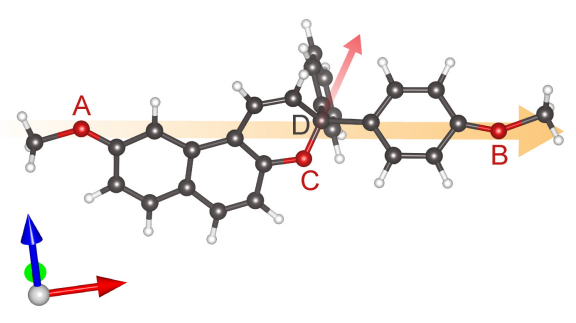

Atom (Coordinates $(\mathrm{x}, \mathrm{y}, \mathrm{z})$ )

A $(-7.229,-0.340,1.454)$

B $(7.254,-1.410,-0.370)$

C $(0.355,-0.735,-0.669)$

D $(1.156,-0.293,0.489)$

$\angle$ between $\overrightarrow{\mathrm{AB}}$ and $\overrightarrow{\mathrm{CD}}=65^{\circ}$

Figure S5. Geometrical evaluation of the alignment between the externally applied force and the C-O pyran bond as a function of naphthopyran regiochemistry.

The change in length between the two attachment points of a molecule that accompanies a covalent transformation offers an alternative description of mechanochemical coupling. ${ }^{9}$ Although mechanochemical ring-opening is only predicted and experimentally observed for the naphthopyran regioisomer substituted at the 5-position, the hypothetical elongation of each naphthopyran regioisomer upon ring-opening reaction was evaluated. Equilibrium geometries were calculated by DFT (B3LYP/6-31G*) for each theoretical merocyanine regioisomer in the cis configuration and the distance between methyl groups was compared with the calculated equilibrium structure of the corresponding naphthopyran (Figure S6). The results of this analysis, which are consistent with the geometrical alignment analysis, indicate a significantly greater elongation and thus enhanced mechanochemical coupling for the naphthopyran regioisomer substituted at the 5-position. 


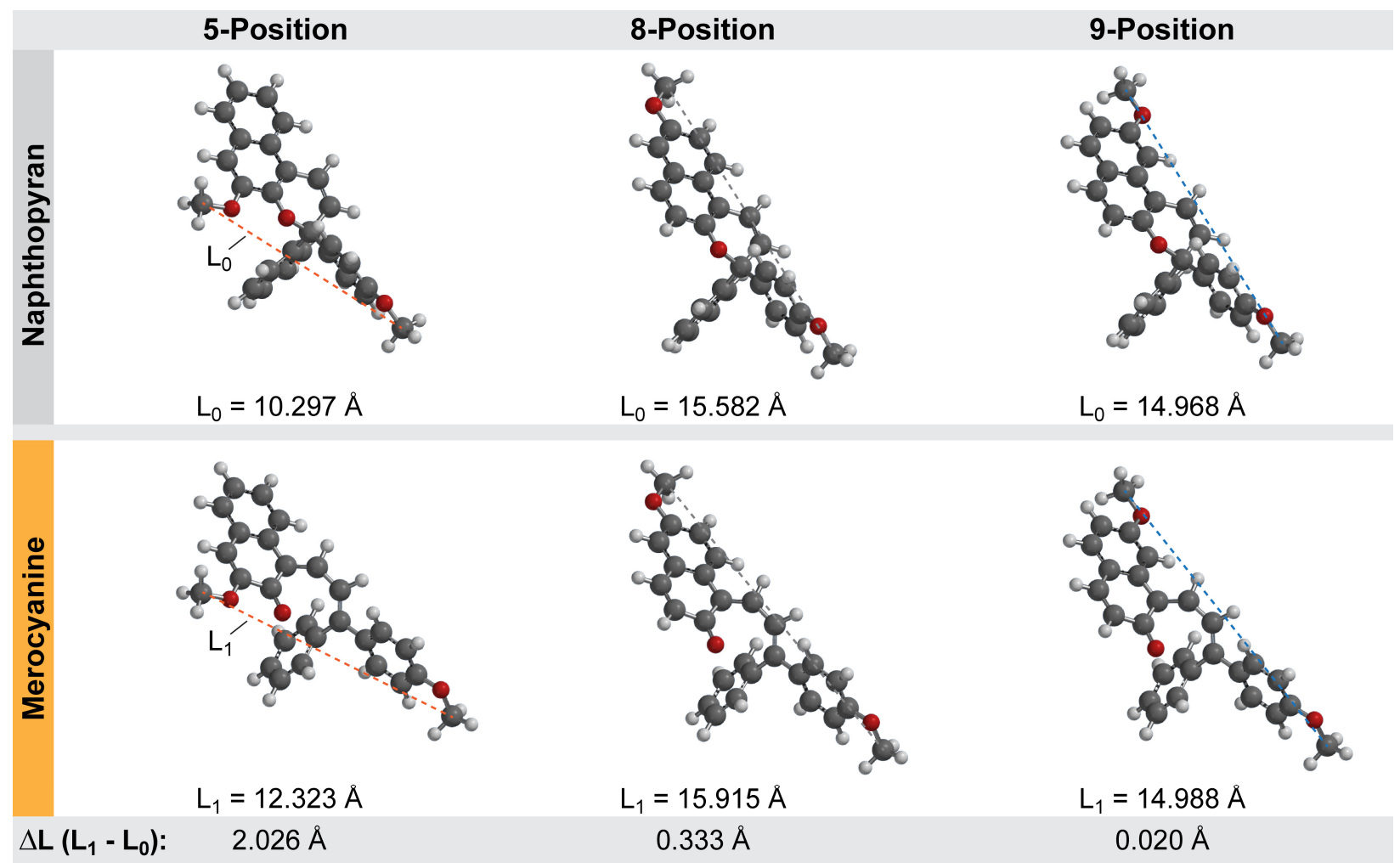

Figure S6. Evaluation of the molecular elongation between naphthopyran and merocyanine forms of each regioisomer. Equilibrium geometries were calculated by DFT at the B3LYP/6-31G* level of theory. The distances between methyl carbon atoms $\left(L_{0}\right.$ and $\left.L_{1}\right)$ are illustrated.

\section{References}

(1) Gabbutt, C. D.; Heron, B. M.; Instone, A. C.; Thomas, D. A.; Partington, S. M.; Hursthouse, M. B.; Gelbrich, T. Eur. J. Org. Chem. 2003, 1220-1230.

(2) Gabbutt, C. D.; Heron, B. M.; Instone, A. C. Heterocycles 2003, 60, 843-855.

(3) Bolchi, C.; Catalano, P.; Fumagalli, L.; Gobbi, M.; Pallavicini, M.; Pedretti, A.; Villa, L.; Vistoli, G.; Valoti, E. Bioorg. Med. Chem. 2004, 12, 4937-4951.

(4) Gossweiler, G. R.; Hewage, G. B.; Soriano, G.; Wang, Q.; Welshofer, G. W.; Zhao, X.; Craig, S. L. ACS Macro Lett. 2014, 3, 216-219.

(5) Beyer, M. K. J. Chem. Phys. 2000, 112, 7307-7312.

(6) Davis, D. A.; Hamilton, A.; Yang, J.; Cremar, L. D.; Van Gough, D.; Potisek, S. L.; Ong, M. T.; Braun, P. V.; Martínez, T. J.; White, S. R.; Moore, J. S.; Sottos, N. R. Nature 2009, 459, 68-72.

(7) Kryger, M. J.; Munaretto, A. M.; Moore, J. S. J. Am. Chem. Soc. 2011, 133, 18992-18998.

(8) Momma, K.; Izumi, F. J. Appl. Cryst. 2011, 44, 1272-1276.

(9) Brown, C. L.; Craig, S. L. Chem. Sci. 2015, 6, 2158-2165. 


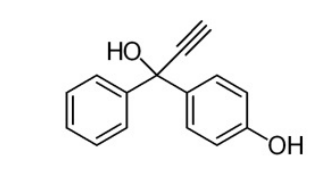

${ }^{1} \mathrm{H}\left(500 \mathrm{MHz}\right.$, acetone- $\left.d_{6}\right)$
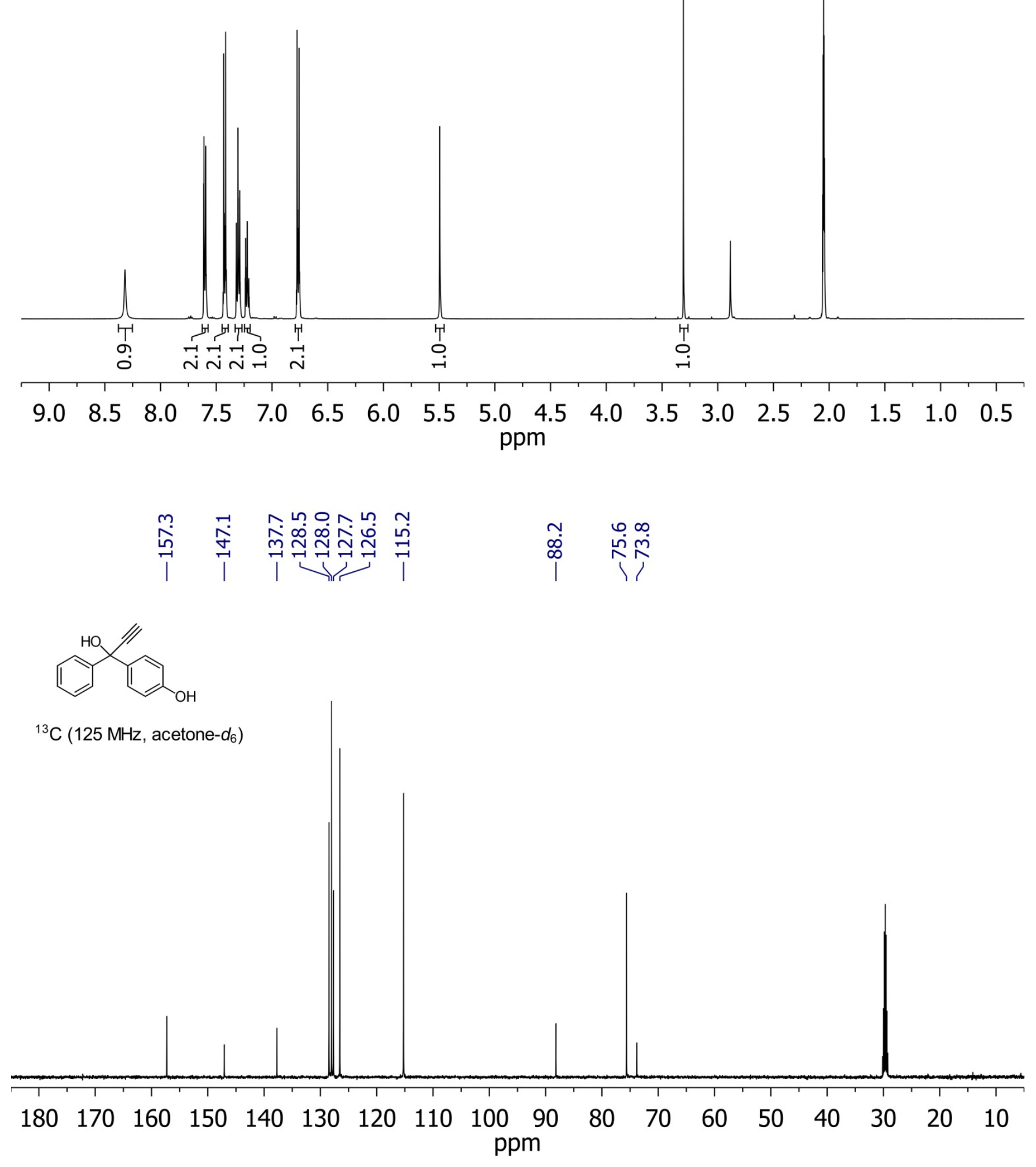

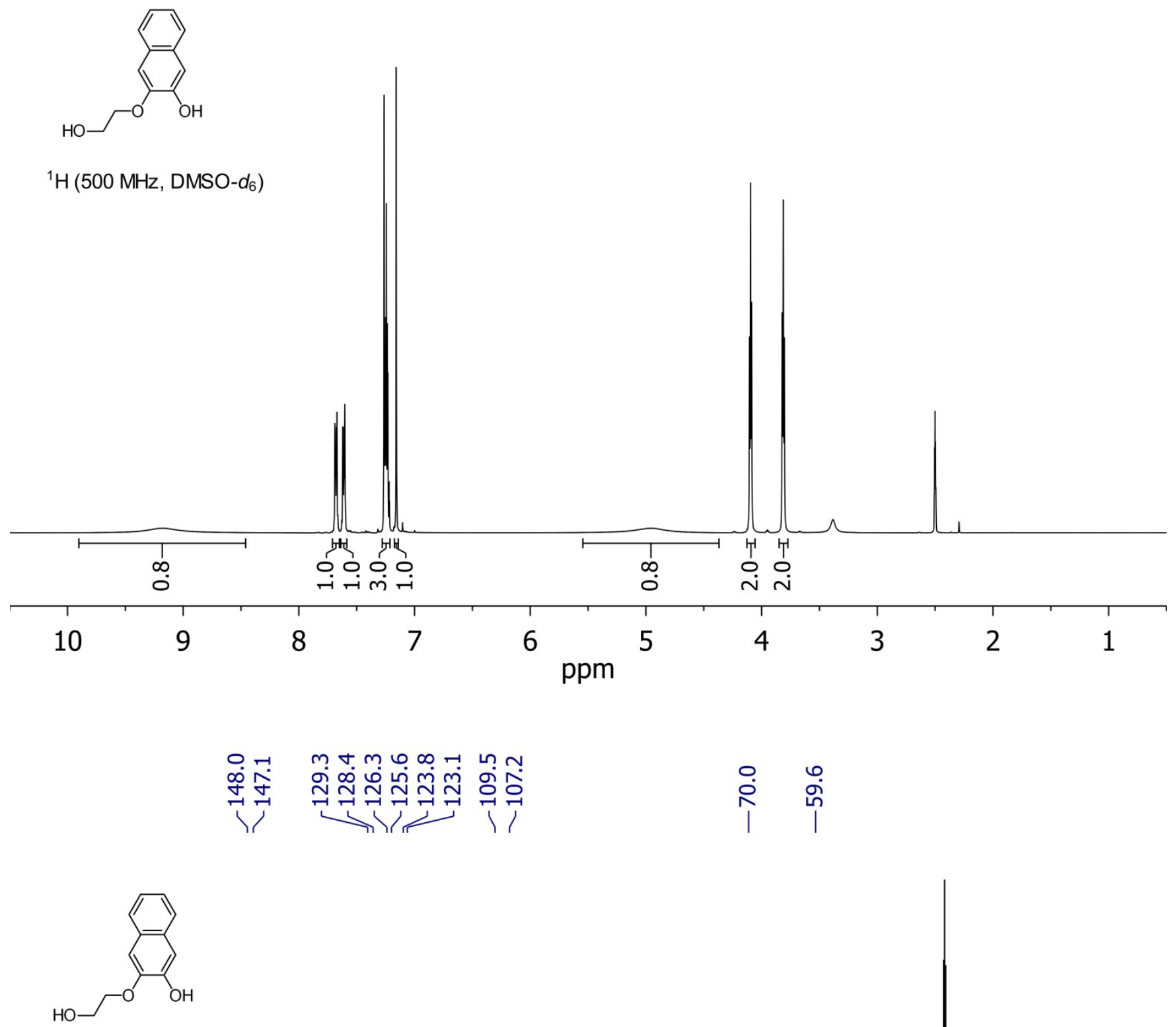

${ }^{13} \mathrm{C}\left(125 \mathrm{MHz}\right.$, DMSO- $\left.d_{6}\right)$

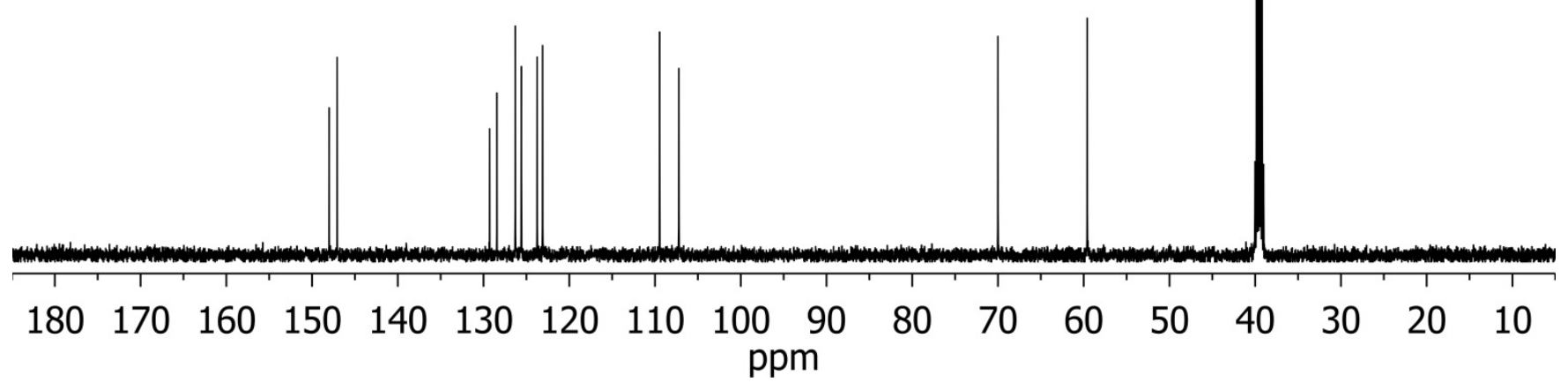



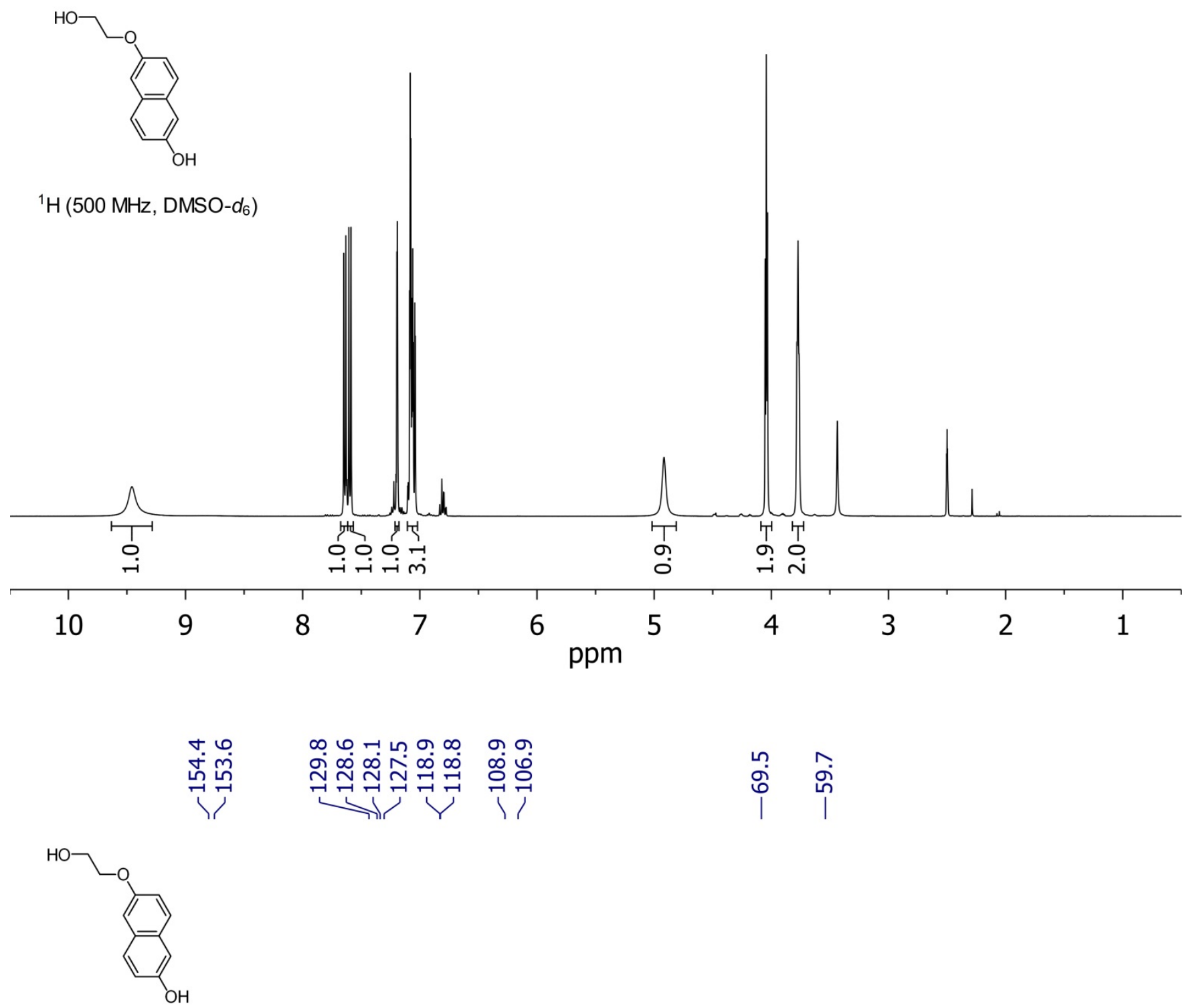

${ }^{13} \mathrm{C}\left(125 \mathrm{MHz}\right.$, DMSO- $\left.d_{6}\right)$

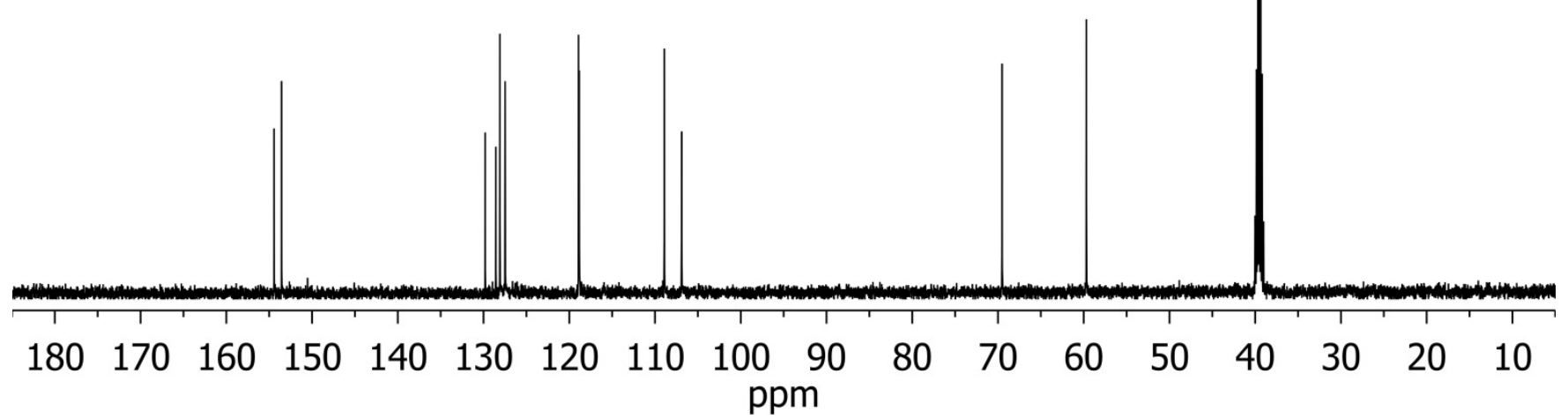




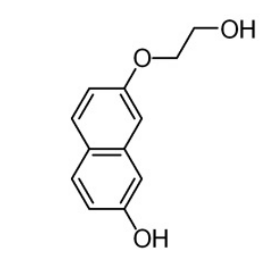

${ }^{1} \mathrm{H}\left(500 \mathrm{MHz}\right.$, acetone- $\left.d_{6}\right)$
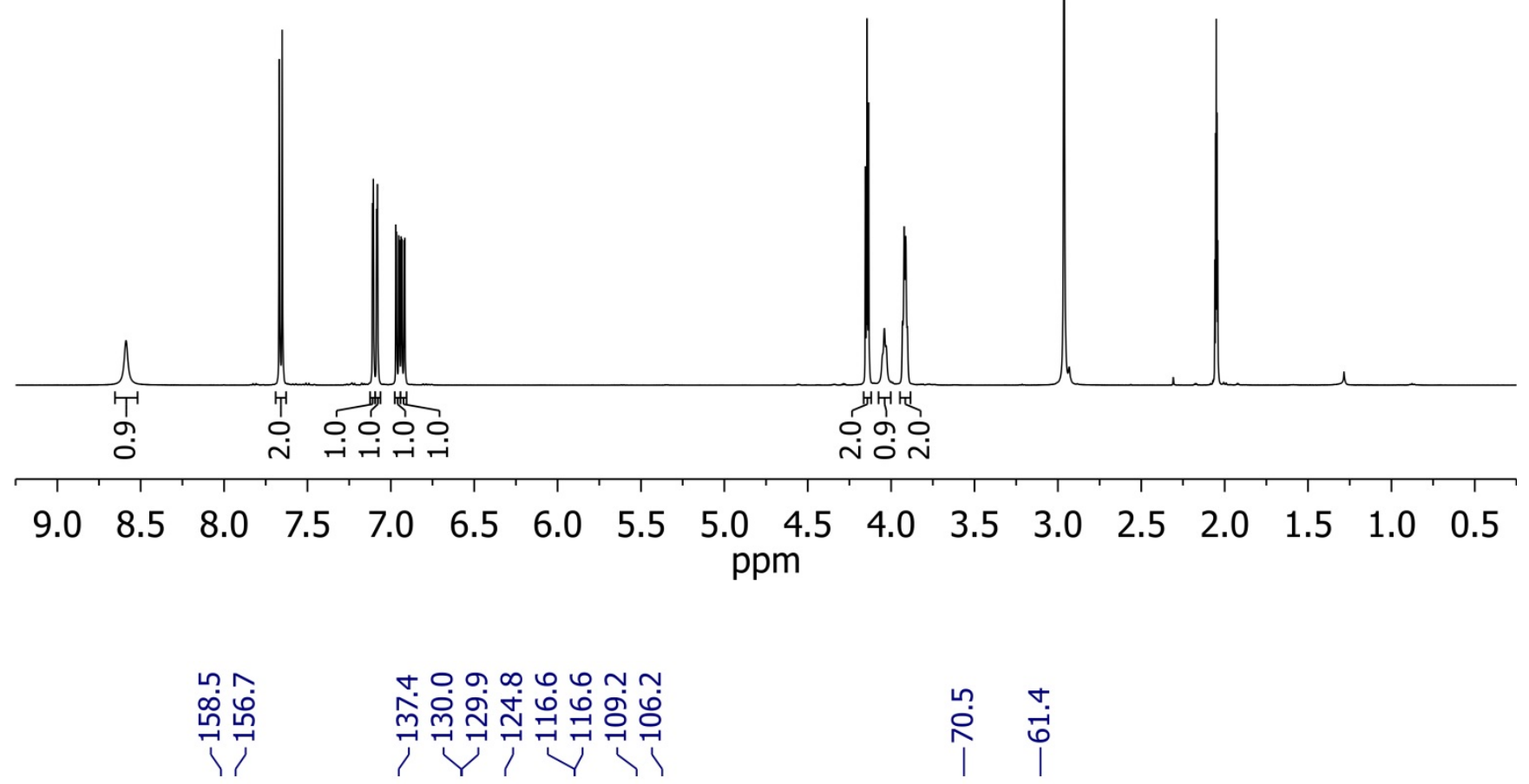

$\overbrace{1}^{\infty}$

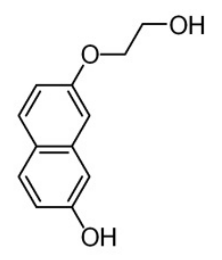

${ }^{13} \mathrm{C}\left(125 \mathrm{MHz}\right.$, acetone- $\left.d_{6}\right)$

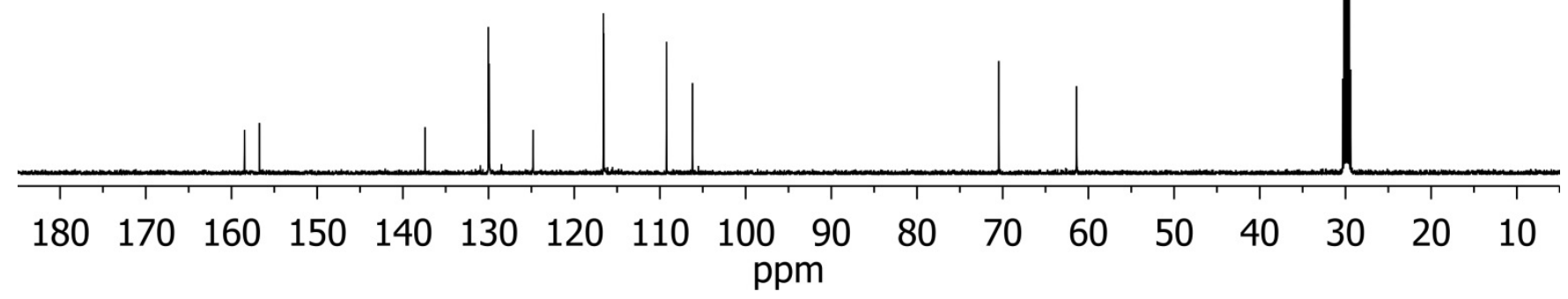



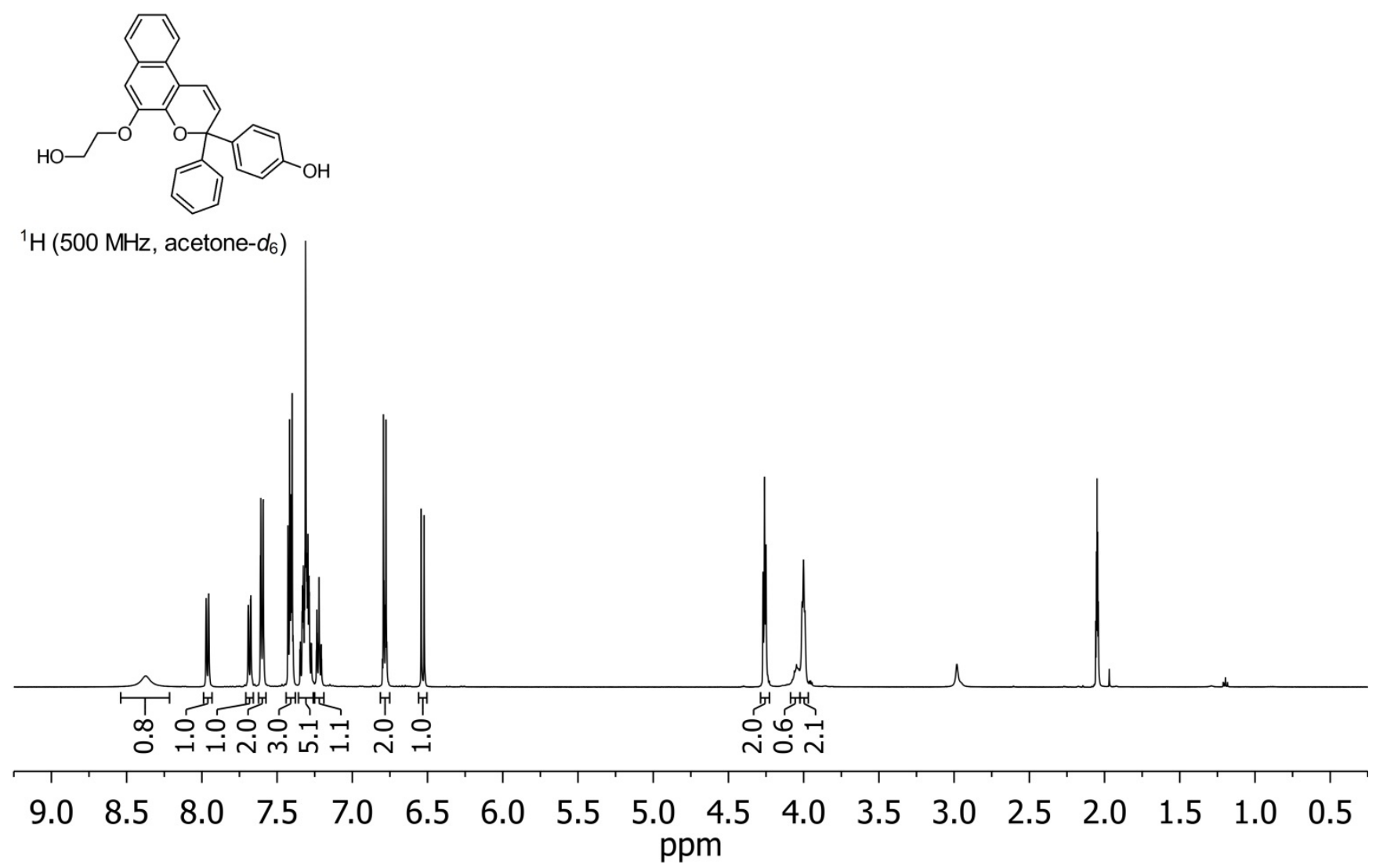

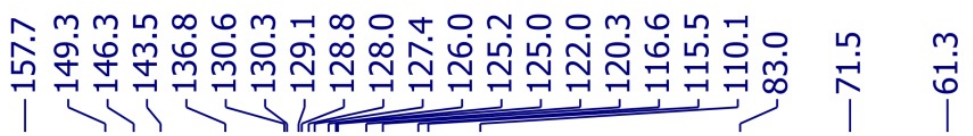

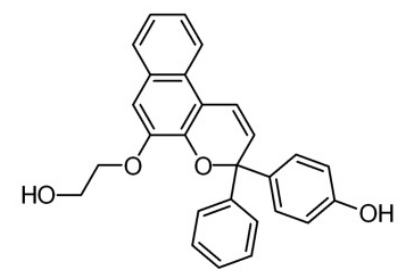

${ }^{13} \mathrm{C}\left(125 \mathrm{MHz}\right.$, acetone- $\left.d_{6}\right)$

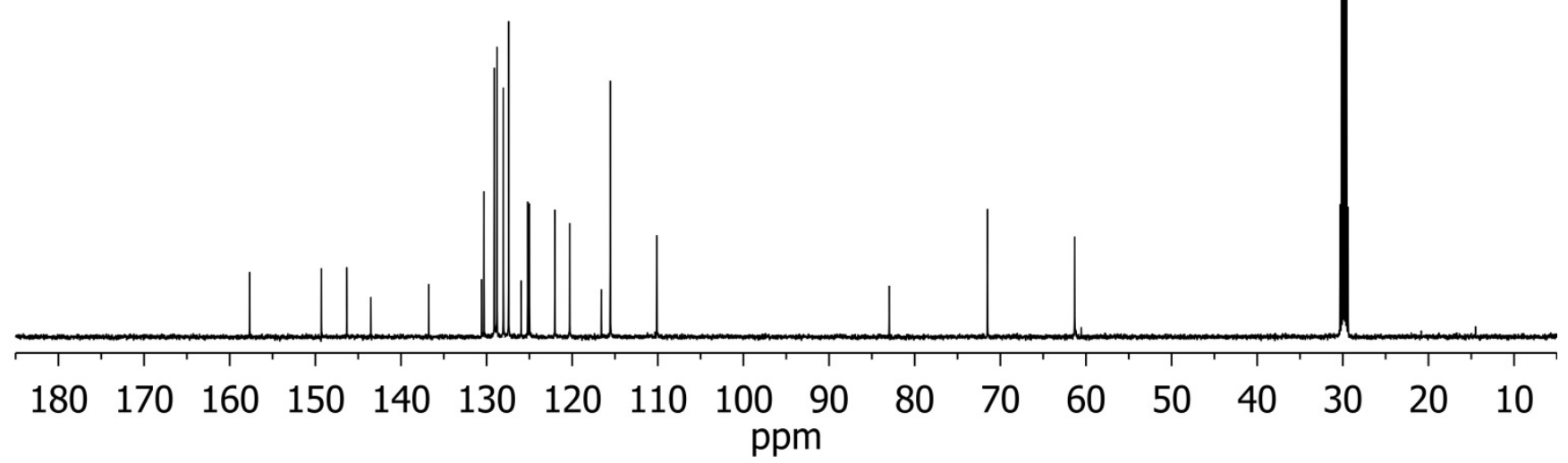



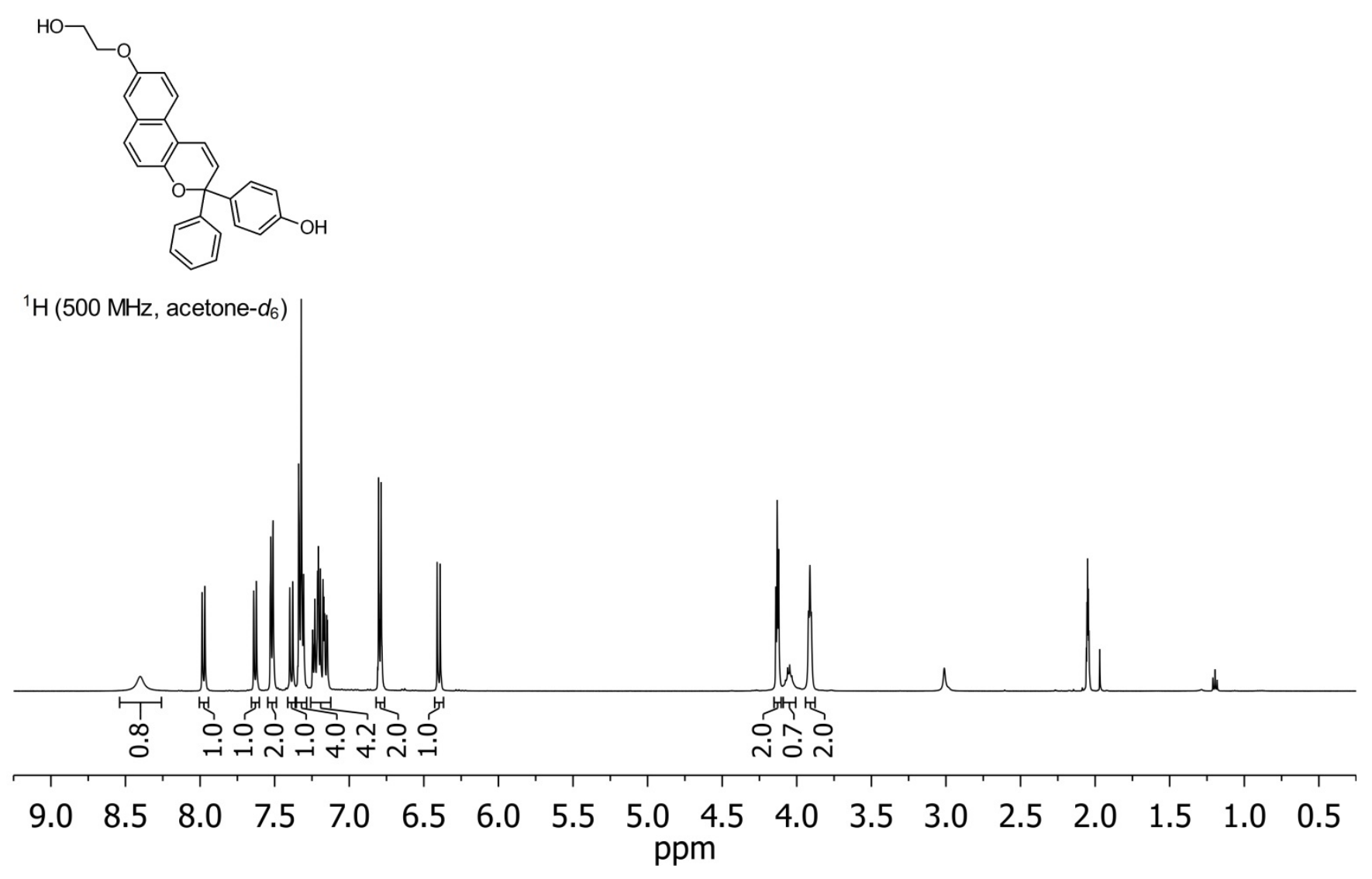

ம

|

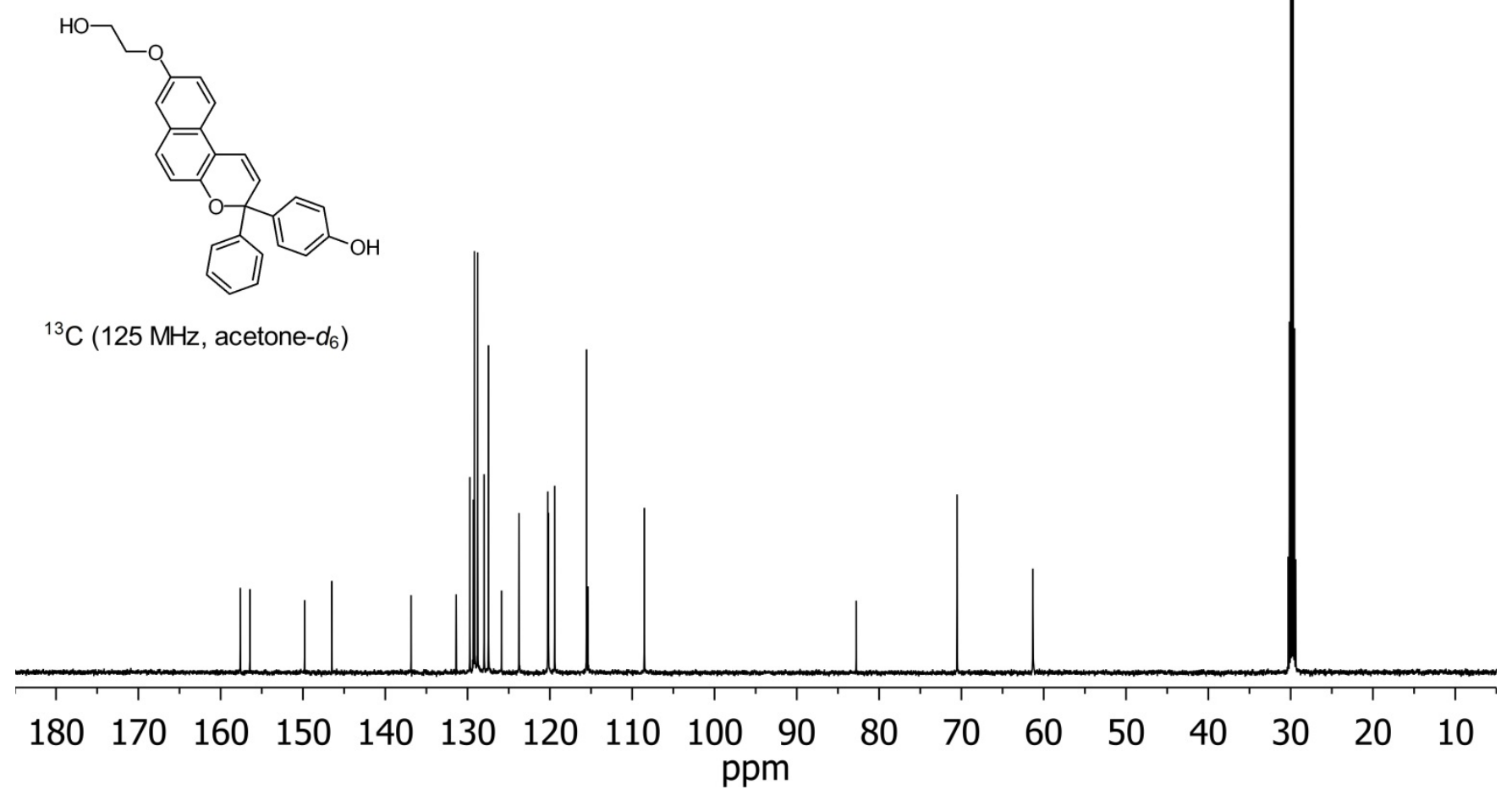



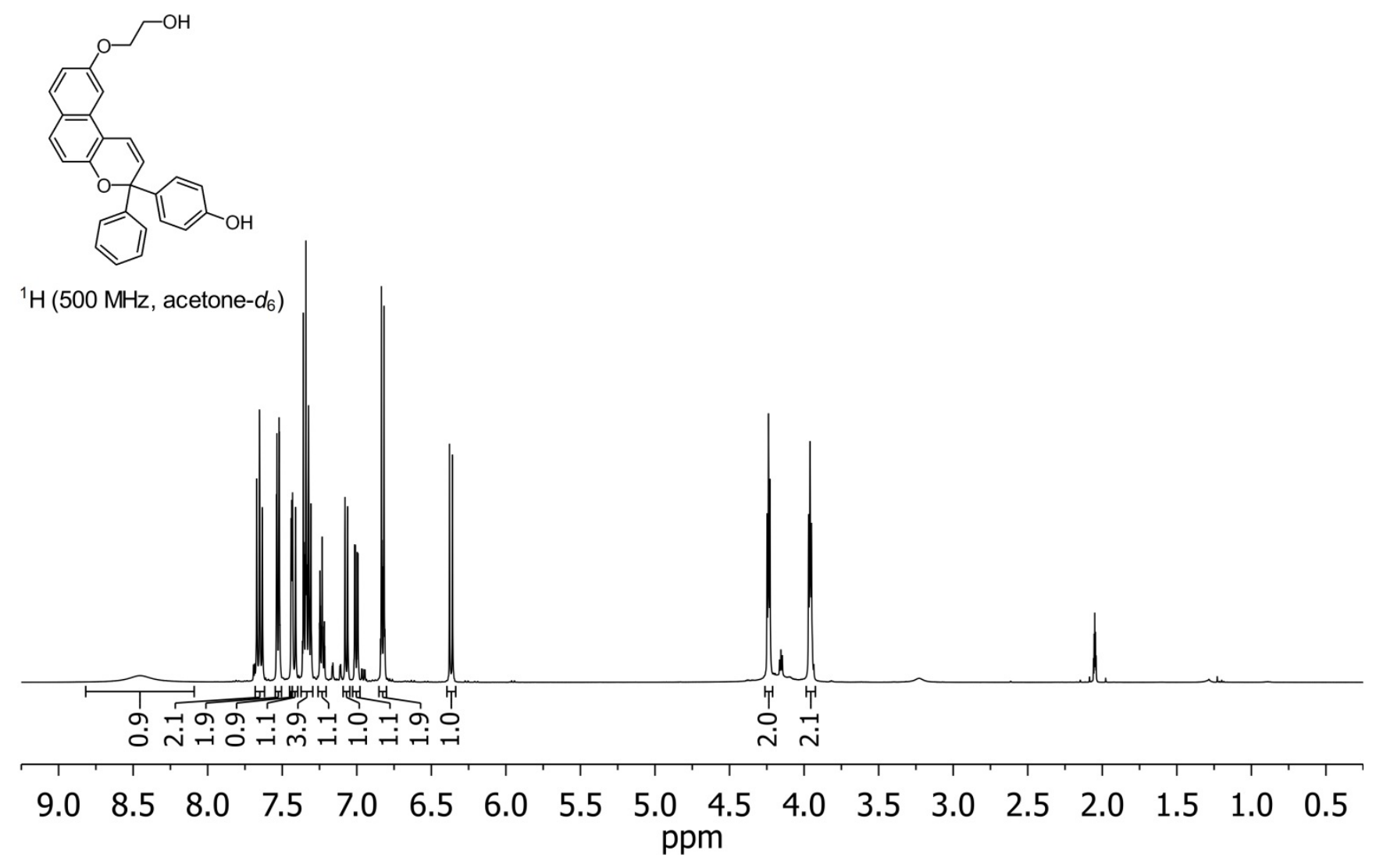

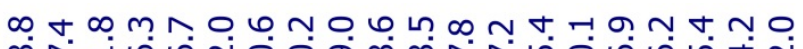
究 望 กิ

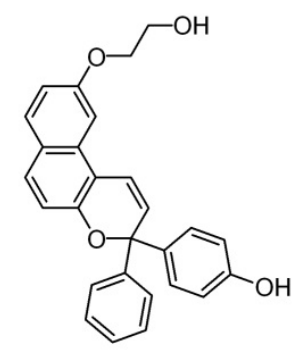

${ }^{13} \mathrm{C}\left(125 \mathrm{MHz}\right.$, acetone- $\left.d_{6}\right)$

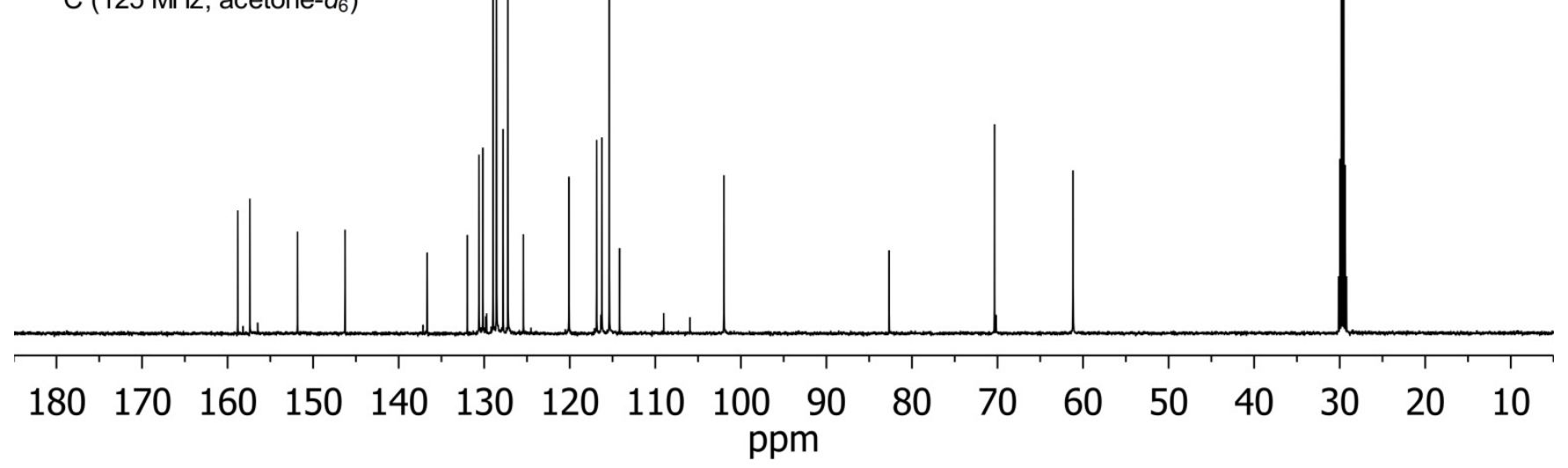




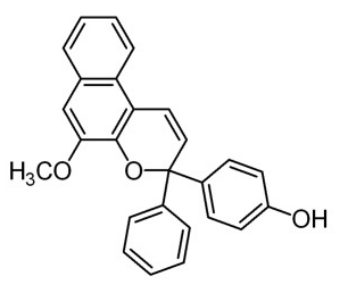

${ }^{1} \mathrm{H}\left(500 \mathrm{MHz}\right.$, acetone- $\left.d_{6}\right)$

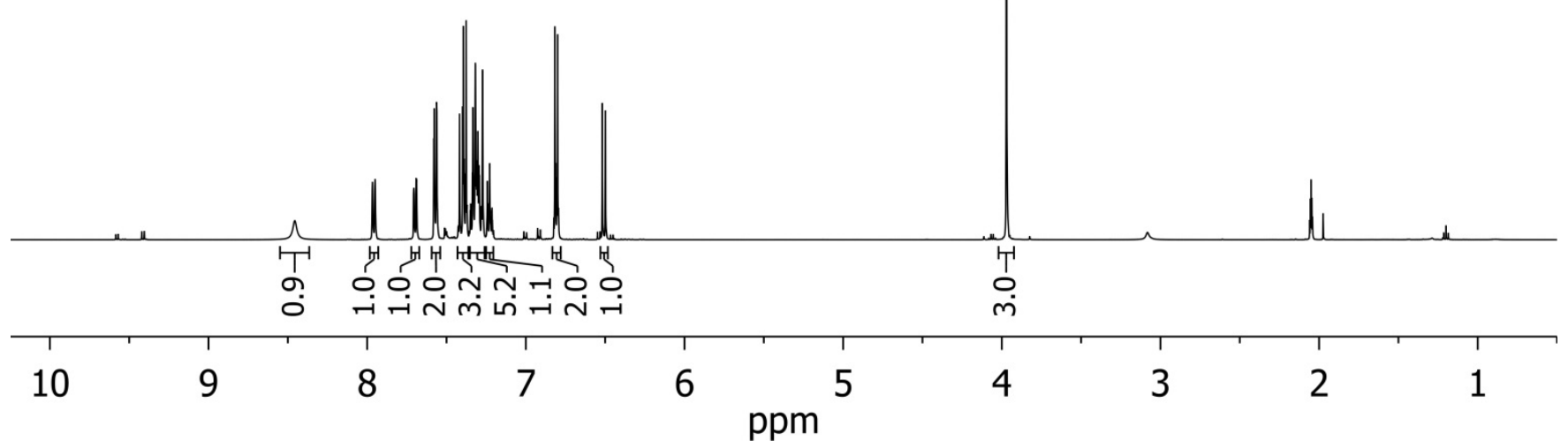

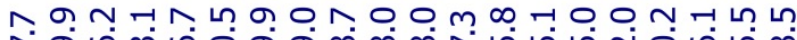
กิ่

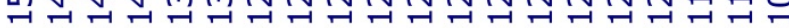

m

ตี่

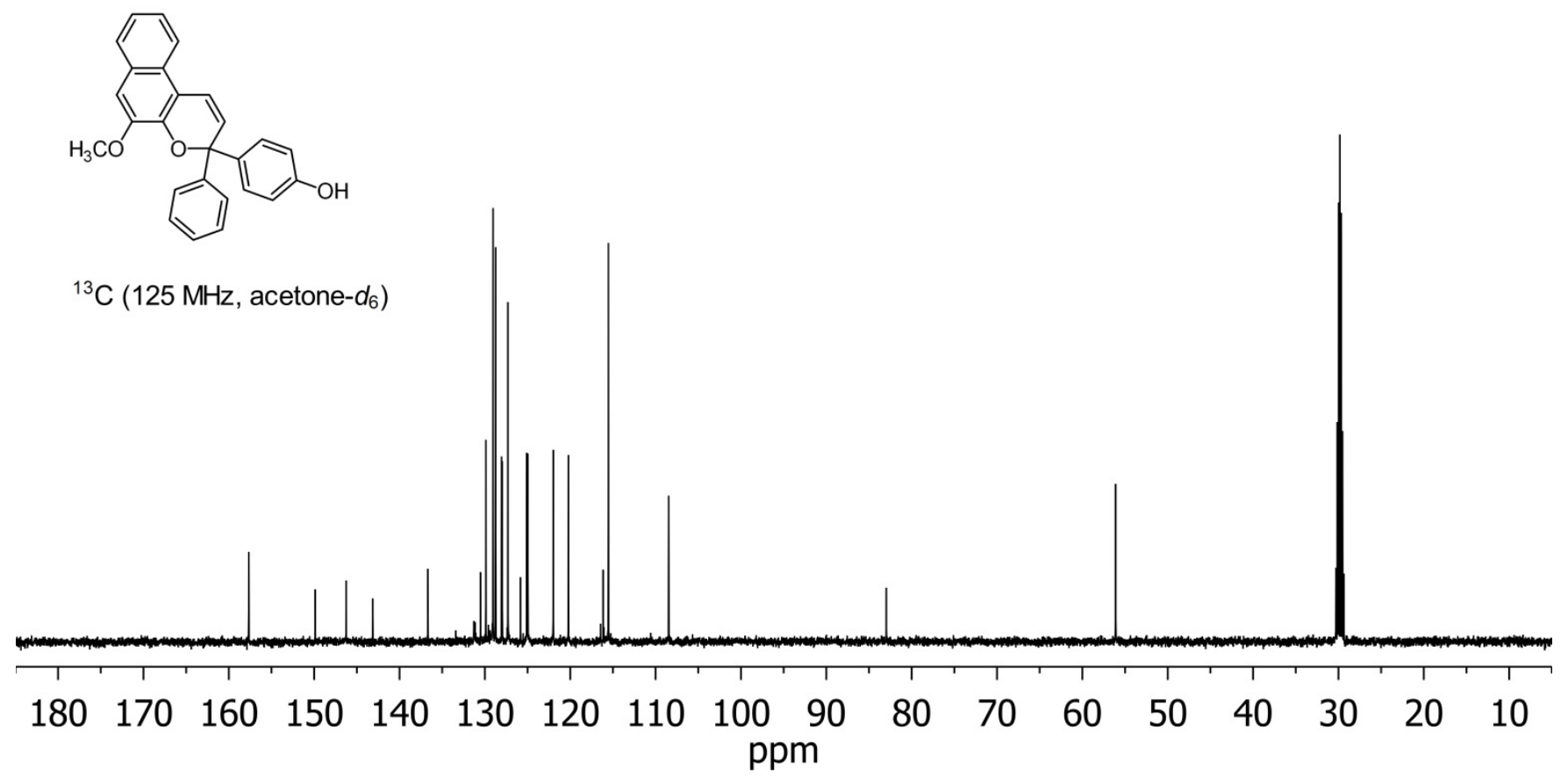




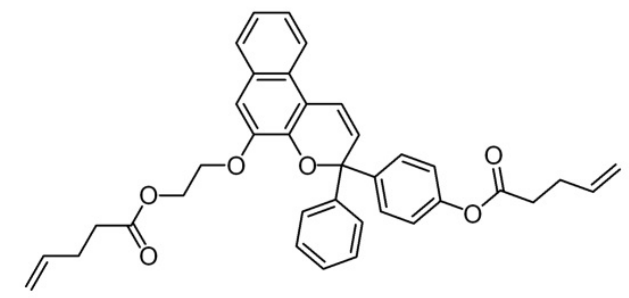

${ }^{1} \mathrm{H}\left(500 \mathrm{MHz}\right.$, acetone- $\left.d_{6}\right)$

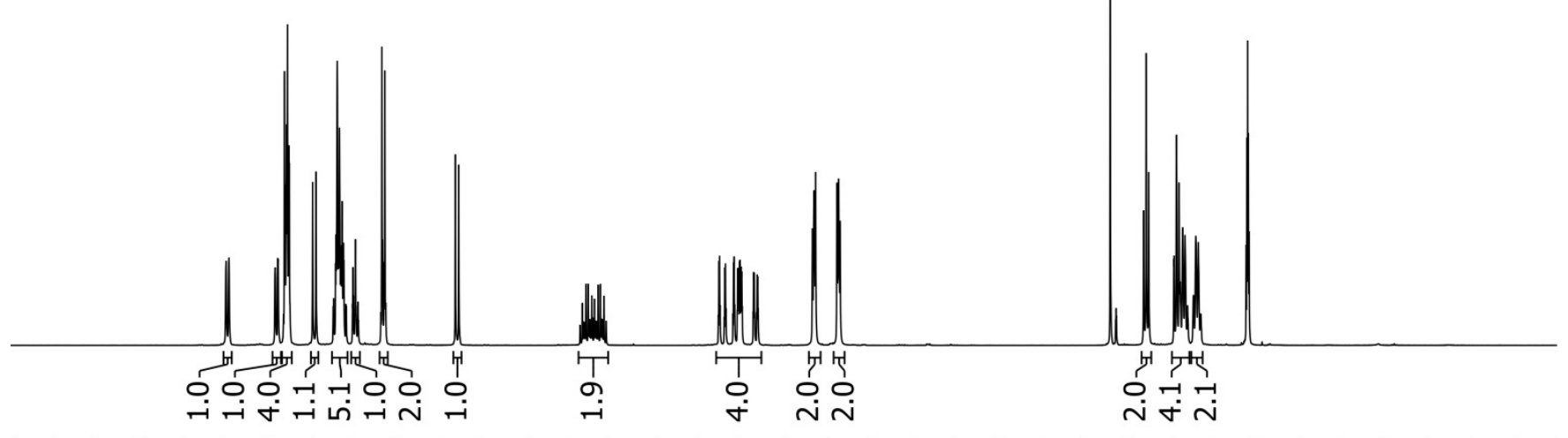

\section{$\begin{array}{llllllllllllllllll}9.0 & 8.5 & 8.0 & 7.5 & 7.0 & 6.5 & 6.0 & 5.5 & 5.0 & 4.5 & 4.0 & 3.5 & 3.0 & 2.5 & 2.0 & 1.5 & 1.0 & 0.5\end{array}$} ppm

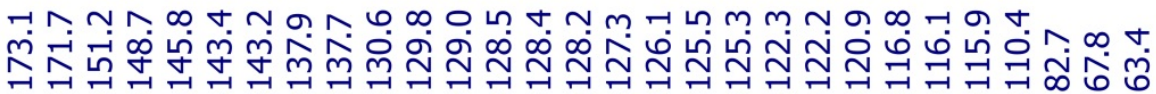

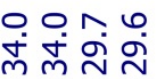

1

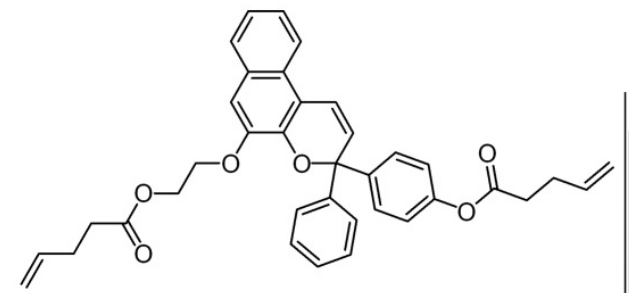

${ }^{13} \mathrm{C}\left(125 \mathrm{MHz}\right.$, acetone- $\left.d_{6}\right)$
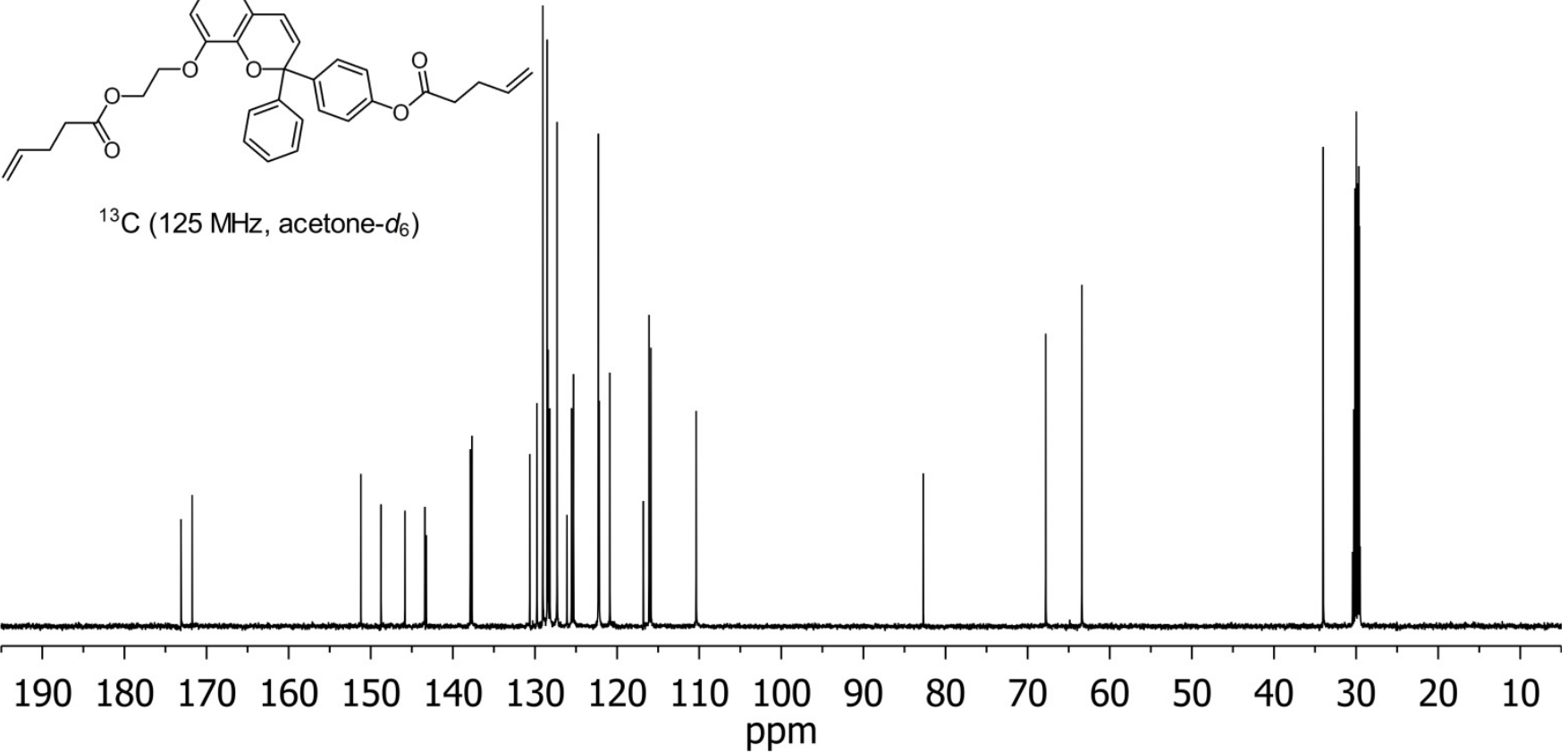


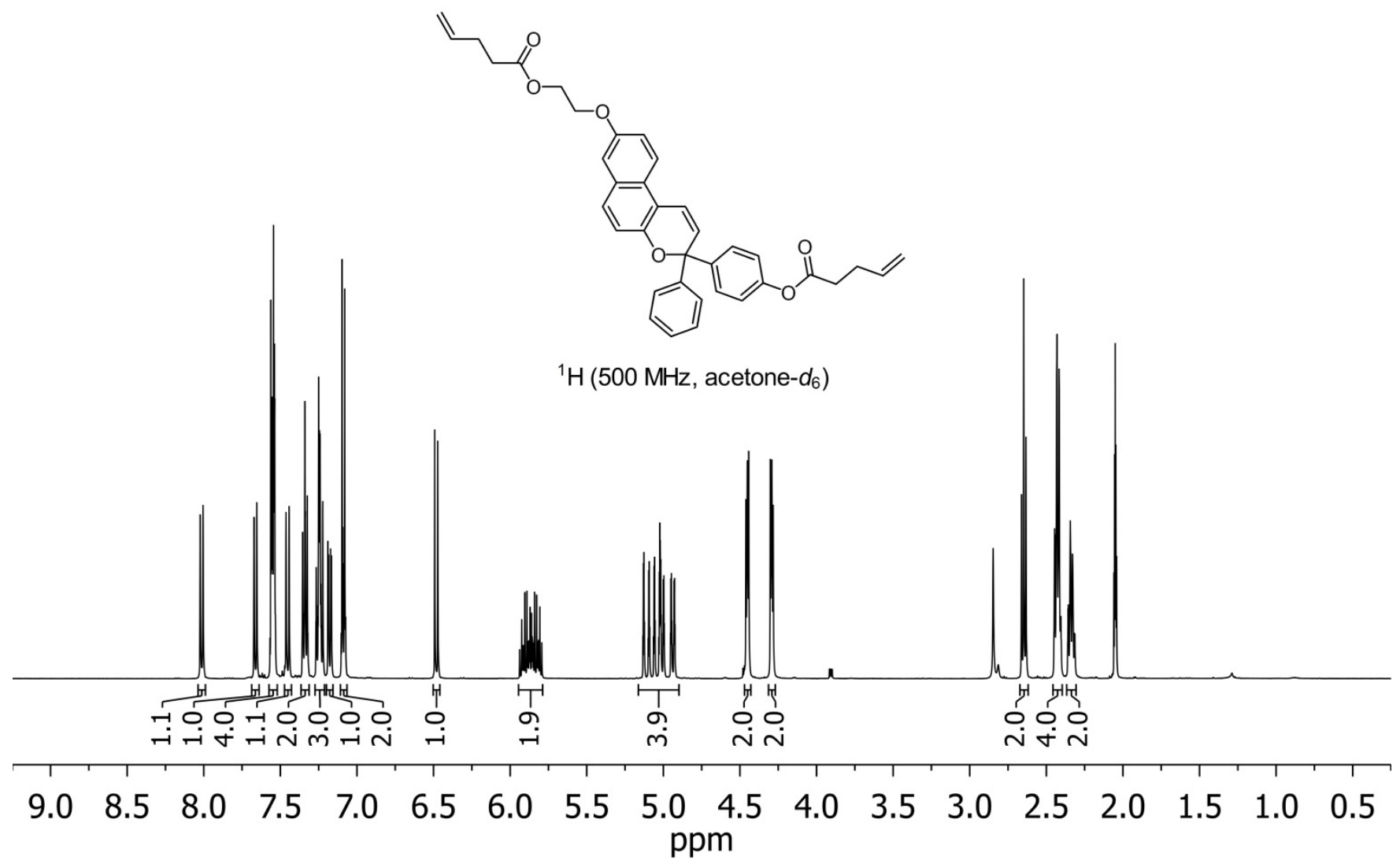

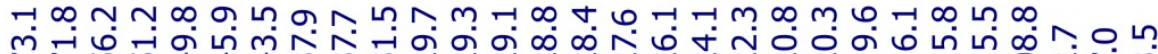
쓱슴

L

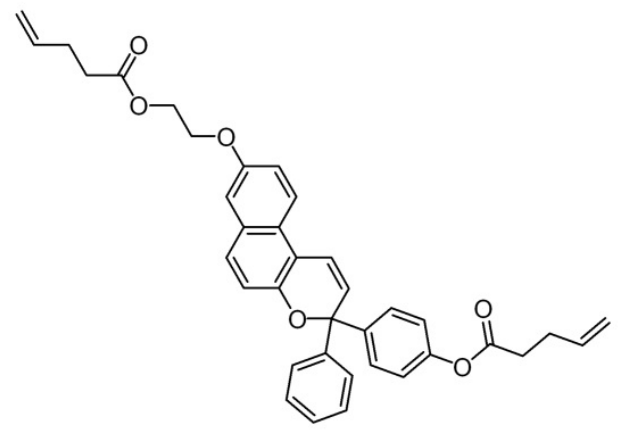

${ }^{13} \mathrm{C}\left(125 \mathrm{MHz}\right.$, acetone- $\left.d_{6}\right)$

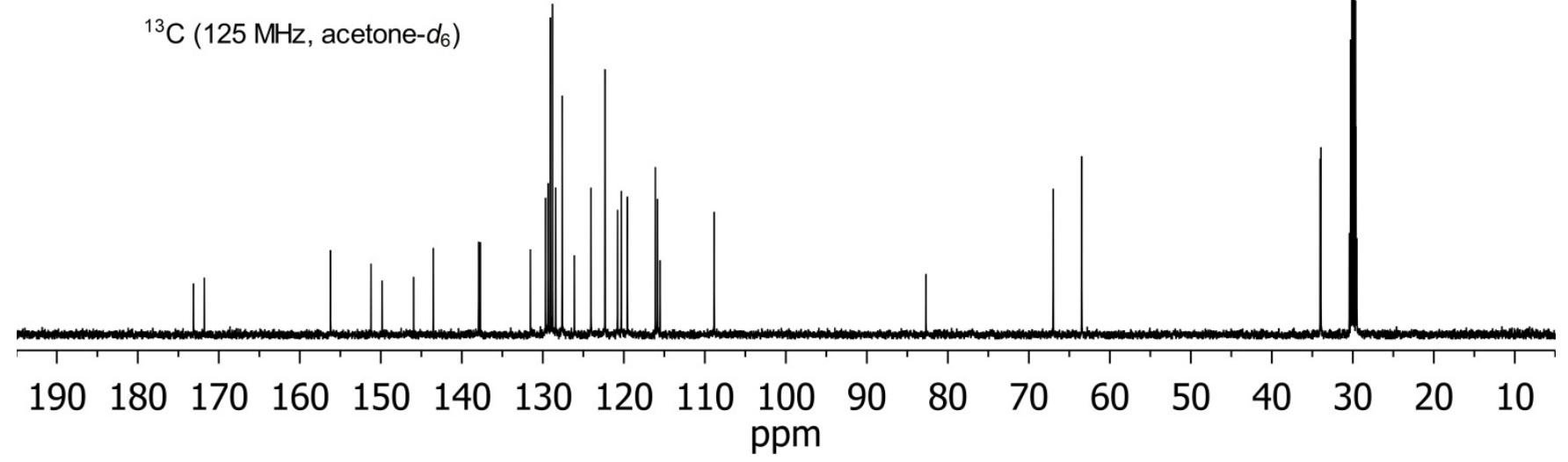




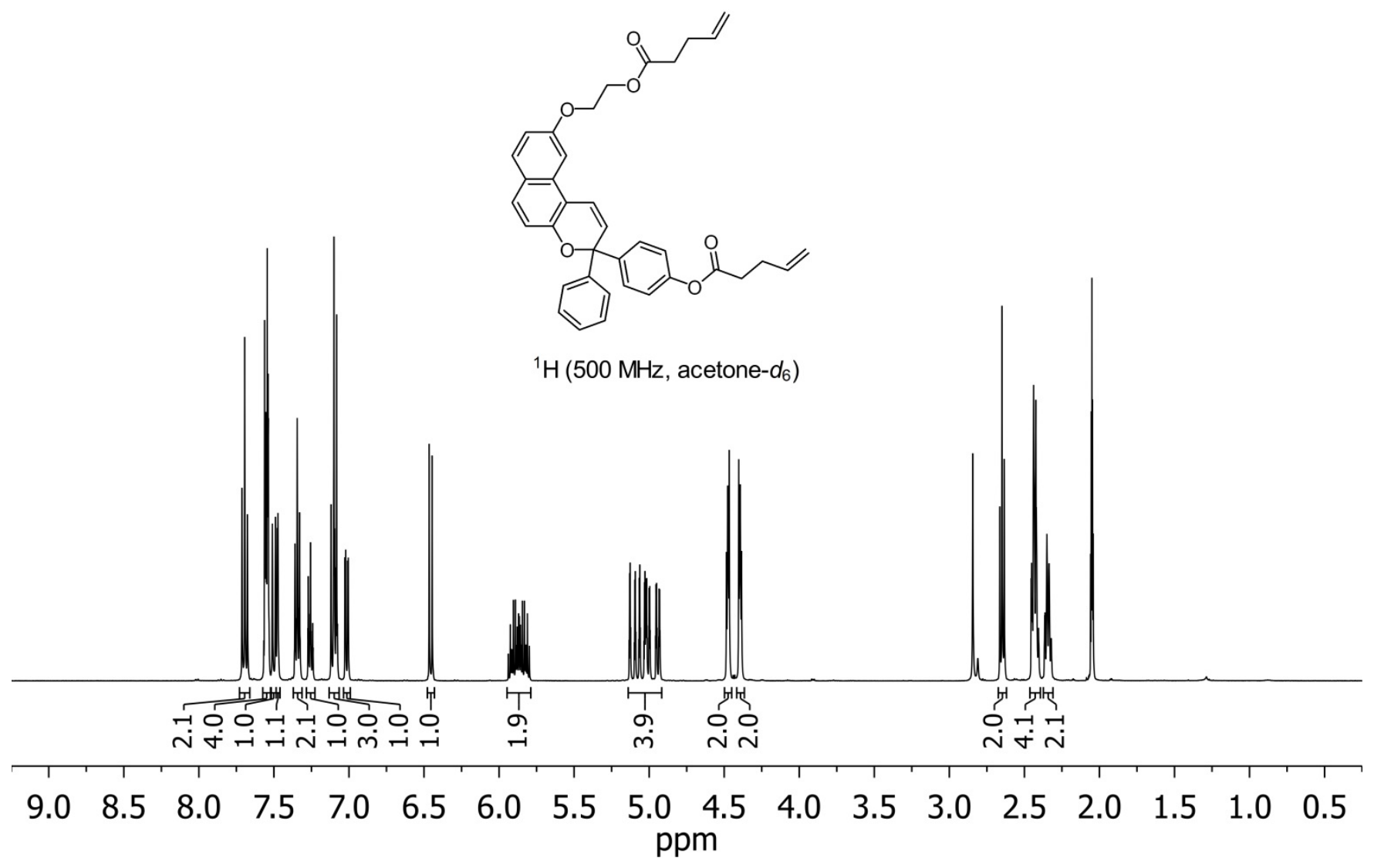

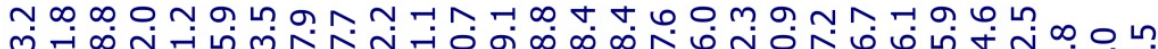

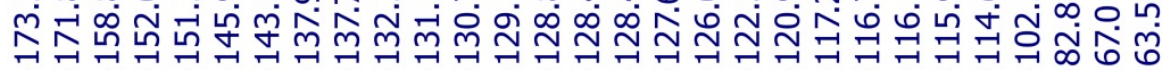

울ํำ

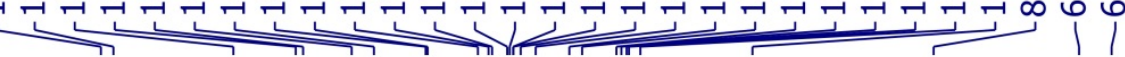

Y

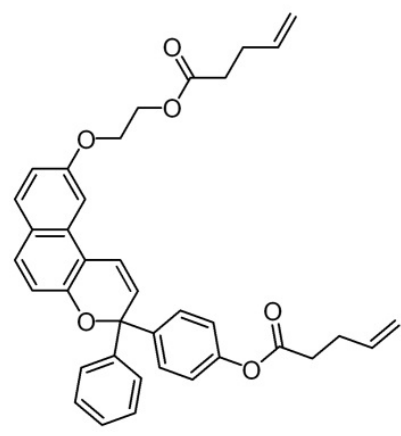

${ }^{13} \mathrm{C}\left(125 \mathrm{MHz}\right.$, acetone- $\left.d_{6}\right)$

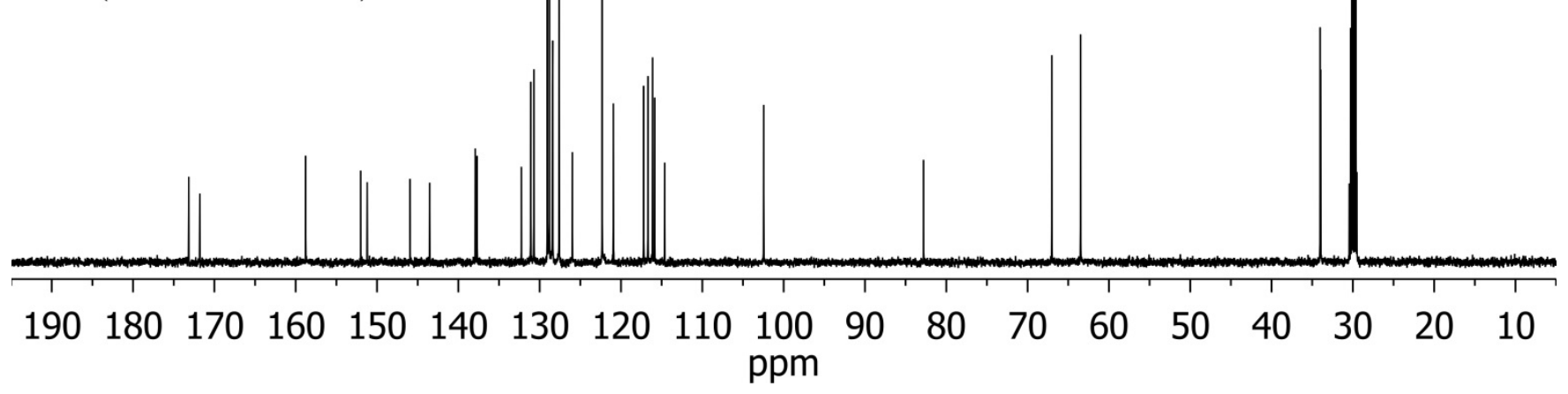




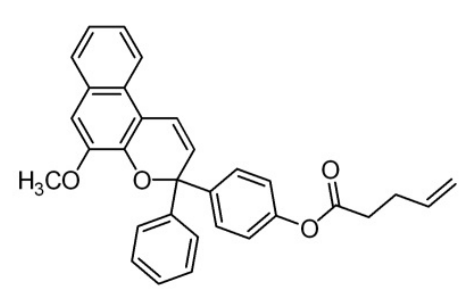

${ }^{1} \mathrm{H}\left(500 \mathrm{MHz}\right.$, acetone- $\left.d_{6}\right)$

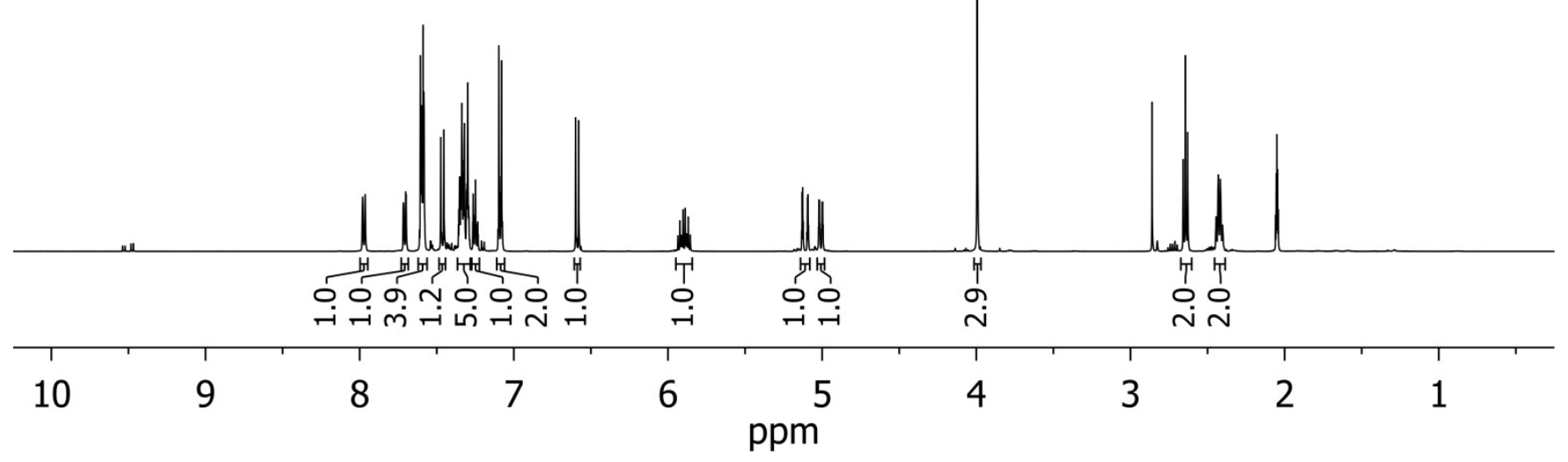

$\infty$ m

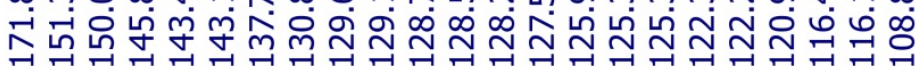

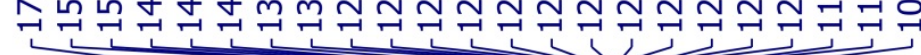

\section{๓)}

ตุ

ํํำ

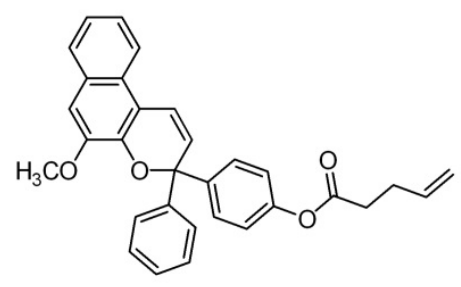

${ }^{13} \mathrm{C}\left(125 \mathrm{MHz}\right.$, acetone $\left.-d_{6}\right)$

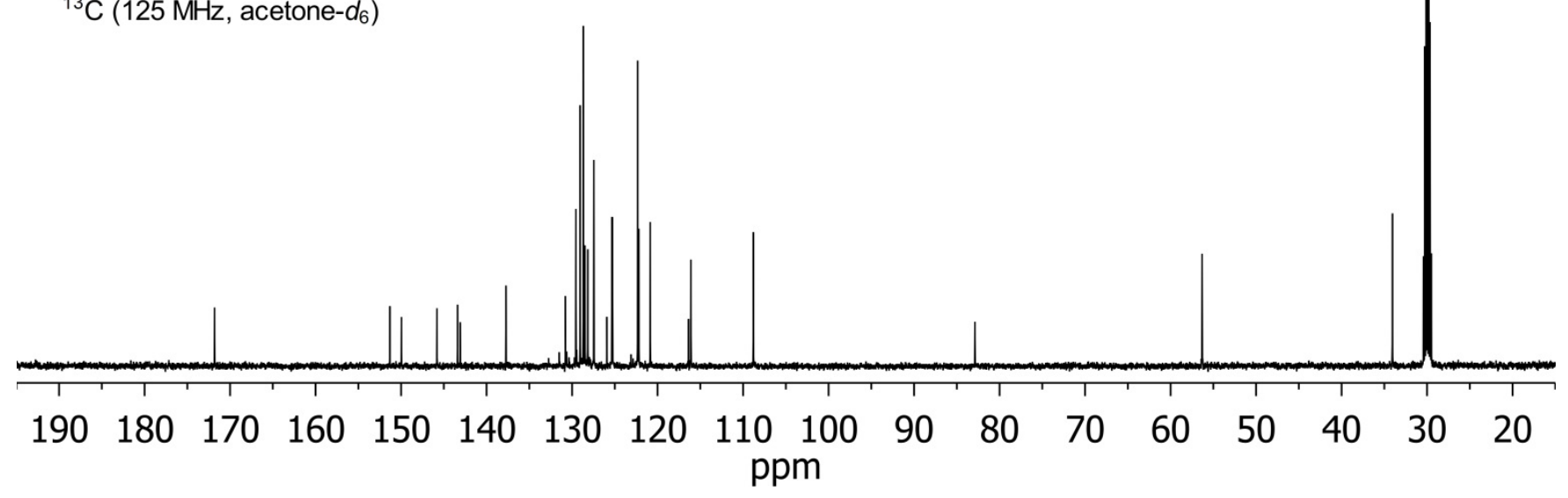

